\title{
An inducible CRISPR-ON system for controllable gene activation in human pluripotent stem cells
}

\author{
Jianying Guo ${ }^{1}$, Dacheng $\mathrm{Ma}^{2}$, Rujin Huang ${ }^{1}$, Jia Ming ${ }^{1}$, Min $\mathrm{Ye}^{1}$, Kehkooi Kee ${ }^{1}$, Zhen $\mathrm{Xie}^{2}$, Jie $\mathrm{Na}^{1 凶}$ \\ ${ }^{1}$ Department of Basic Medical Sciences, School of Medicine, Center for Stem Cell Biology, Tsinghua University, Beijing \\ 100084, China \\ 2 MOE Key Laboratory of Bioinformatics and Bioinformatics Division, Center for Synthetic and System Biology, TNLIST/ \\ Department of Automation, Tsinghua University, Beijing 100084, China \\ $\triangle$ Correspondence: jie.na@tsinghua.edu.cn (J. Na)
}

Received September 13, 2016 Accepted December 1, 2016

\begin{abstract}
Human pluripotent stem cells (hPSCs) are an important system to study early human development, model human diseases, and develop cell replacement therapies. However, genetic manipulation of hPSCs is challenging and a method to simultaneously activate multiple genomic sites in a controllable manner is sorely needed. Here, we constructed a CRISPR-ON system to efficiently upregulate endogenous genes in hPSCs. A doxycycline (Dox) inducible dCas9-VP64-p65-Rta (dCas9-VPR) transcription activator and a reverse Tet transactivator (rtTA) expression cassette were knocked into the two alleles of the AAVS1 locus to generate an iVPR hESC line. We showed that the dCas9-VPR level could be precisely and reversibly controlled by the addition and withdrawal of Dox. Upon transfection of multiplexed gRNA plasmid targeting the NANOG promoter and Dox induction, we were able to control NANOG gene expression from its endogenous locus. Interestingly, an elevated NANOG level promoted naïve pluripotent gene expression, enhanced cell survival and clonogenicity, and enabled hESCs to integrate with the inner cell mass (ICM) of mouse blastocysts in vitro. Thus, iVPR cells provide a convenient platform for gene function studies as well as high-throughput screens in hPSCs.
\end{abstract}

Electronic supplementary material The online version of this article (doi:10.1007/s13238-016-0360-8) contains supplementary material, which is available to authorized users.
KEYWORDS CRISPR, transcription activation, human pluripotent stem cells, NANOG, pluripotency

\section{INTRODUCTION}

Human pluripotent stem cells (hPSCs), including human embryonic stem cells (hESCs) and human induced pluripotent stem cells (hiPSCs), are capable of self-renewal indefinitely and have the potential to differentiate into all cell types in the human body. Therefore this system offers a useful platform to study early human embryogenesis and a potential cell source for regenerative medicine. Moreover, functional cells derived from hESCs can be used to model human diseases in the context of drug toxicity tests and new drug development. These applications rely on methods to precisely control gene expression. However, because of difficulties in culture and transfection, targeted regulation of gene expression in hPSCs remains a technically challenging task. A method for efficient, rapid, and controllable gene activation is sorely needed.

Recently, the clustered regularly interspaced short palindromic repeat (CRISPR)/Cas9 system emerged as a powerful and versatile tool for genome editing (Wiedenheft et al., 2012). CRISPR was initially discovered as the adaptive immune system of bacteria and archaea (Wiedenheft et al., 2012). In response to viral and plasmid infection, bacteria and archaea could cut and degrade the foreign DNA recognized by a matching spacer RNA with the help of the Cas9 enzyme (Wiedenheft et al., 2012). CRISPR was rapidly transformed to a genome editing tool, and it has been shown to work in a wide range of systems, from plants to human cells, since the Cas9 nuclease can be directed easily to 
virtually anywhere in the genome using a short guide RNA and cutting the target DNA (Hsu et al., 2014). In pluripotent stem cells, the CRISPR system has been used to perform highly efficient gene knock-out and knock-in studies (Hsu et al., 2014). In addition to genome editing, a nuclease inactivated Cas9 (dCas9) was developed (Gilbert et al., 2014). By fusing dCas9 with transcription activators and repressors, such as VP64, and KRAB (Balboa et al., 2015; Gilbert et al., 2014; Mandegar et al., 2016; Genga et al., 2016), or with epigenetic modifiers, such as the catalytic domain of acetyltransferase p300 (Hilton et al., 2015) and Tet (ten eleven translocation) dioxygenase (Xu et al., 2016), one can use the CRISPR system to activate or inhibit gene expression or modify the histone and DNA methylation status at the desired locus.

Because of its potential applications in regenerative medicine, random insertion of foreign DNA into the genome of hPSCs should be avoided, since this may cause harmful mutations. The Adeno-Associated Virus Integration Site 1 (AAVS1) locus resides in the first intron of the PPP1R12C gene and has been used as a safe harbor for transgene integration (Smith et al., 2008; Hockemeyer et al., 2009; Lombardo et al., 2011; Qian et al., 2014; Zhu et al., 2014; Genga et al., 2016). Here we generated an iVPR hESC line by knocking-in the inducible dCas9-VPR system into the two alleles of the AAVS1 locus. Detailed characterization of the iVPR hESC demonstrated that dCas9-VPR protein could be induced by Dox within $12 \mathrm{~h}$ and disappear after Dox withdrawal. An inducible NANOG overexpression line (iNANOG) was established based on the iVPR system. We found a significant increase in NANOG protein after Dox induction. INANOG cells upregulated naïve pluripotency genes and were able to grow for a significant length of time in a naïve state medium containing ERK and GSK3 inhibitors and human LIF. The iVPR system can be a valuable system to control gene expression from endogenous loci and serve as platform for genome wide screens to identify new genes that can regulate stem cell self-renewal and differentiation.

\section{RESULTS}

DCas9-VPR mediated robust ectopic and endogenous gene activation in human cell lines

To construct a robust and tunable gene activation system in hPSCs, we first compared the activation efficiency of dCas9VPR (Chavez et al., 2015) with dCas9-VP64 (Kearns et al., 2014) and the Doxycycline (Dox) inducible Tet-On transactivator (rtTA) (Fig. 1A). We constructed plasmids to express gRNA targeting the TetO sequence (gTetO), and tested the ability of dCas9-VPR + gTetO or dCas9-VP64 + gTetO to activate the synthetic TRE promoter driving enhanced blue fluorescent protein expression (TRE-BFP) in 293FT cells (Fig. 1A). The Tet transactivator (rtTA) was used as positive control (Fig. 1B). DCas9-VPR strongly activated BFP fluorescence, $43.1 \%$ of cells were BFP positive, while in the
rtTA + Dox and dCas9-VP64 groups, only $28.2 \%$ and $5.8 \%$ of cells activated BFP, respectively (Fig. $1 \mathrm{C}$ and 1D). Moreover, dCas9-VPR resulted in the strongest mean BFP fluorescence intensity, indicating that it is the strongest activator among the three (Fig. 1D).

We next tested the dCas9-VPR function in hESCs. DCas9-VPR, gTetO, and TRE-BFP plasmids were cotransfected into H9 hESCs. In another group, rtTA and TREBFP plasmids were co-transfected. FACS analysis showed that nearly $17 \%$ of cells in the dCas9-VPR group turned on BFP, while $24.7 \%$ of cells in the rtTA group were BFP positive after Dox induction, and only $0.6 \%$ of cells exhibited BFP fluorescence without Dox (Fig. 1E). Interestingly, the dCas9VPR group showed the strongest mean fluorescence intensity (Fig. 1F). This is consistent with our result based on 293FT cells and proves that dCas9-VPR is a robust transcription activator, even compared with rtTA. We also tested the activation effect of dCas9-VPR in mouse embryonic stem cells (mESCs) and mouse embryonic fibroblasts (MEFs) and obtained similar results (Fig. S1A and S1B).

We then tested the efficiency of dCas9-VPR to activate normally silenced pluripotency genes in human cells. Two gRNAs targeting the -254 and -144 positions upstream of the transcription start site (TSS) of the pluripotency gene NANOG were selected (Fig. 2A). A GFP-2A-Puromycin resistant gene expression cassette was placed after the gRNA cassette both to monitor the transfection efficiency and for selection (Fig. 2A). NANOG cannot be activated by gNANOG alone or by dCas9-VPR together with the control gTetO. However, introducing gNANOG and dCas9-VPR together could elevate the NANOG transcript level by up to 150 -fold in 293FT cells, indicating that it has a robust gene activation function (Fig. 2C).

Next, we tested whether the dCas9-VPR system could simultaneously activate multiple genes in human cells, we designed 2 different gRNAs per gene promoter for HOXA10, SNAIL1, MESP1, GATA5 and HOXA9. First we tested the activation efficiency of these gRNAs towards their target genes when transfected separately in 293FT cells (Fig. 2D). Q-PCR analysis showed all of the five pairs of gRNAs can activate their target gene upon co-transfection with dCas9VPR (Fig. 2D). We next pooled gRNA pairs of two genes (2× gRNAs: MESP1, GATA5), three genes ( $3 \times$ gRNAs: HOXA10, SNAIL1, HOXA9) or five genes (5× gRNAs: HOXA10, SNAIL1, MESP1, GATA5 and HOXA9) to test the co-activation efficiency. Upon co-transfection with dCas9VPR, different combination of gRNAs upregulated their target genes together (Fig. 2E), indicating that dCas9-VPR system could be a useful tool for multiplexed endogenous gene activation.

To validate the utility of the dCas9-VPR system in hESCs, we transfected H9 hESCs with either dCas9-VPR and gNANOG or with rtTA and NANOG coding DNA sequence (CDS) joined to $\mathrm{H} 2 \mathrm{~B}$-mCherry through a $2 \mathrm{~A}$ peptide driven by a TRE promoter. As shown in Fig. 3A, for the dCas9-VPR group, increased NANOG protein expression (in 
A

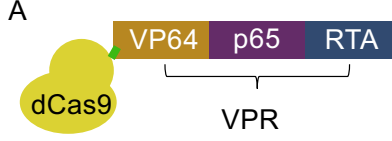

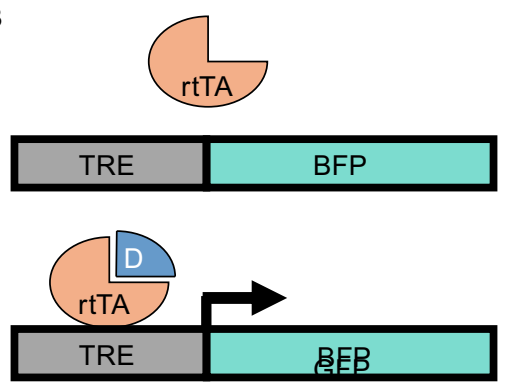

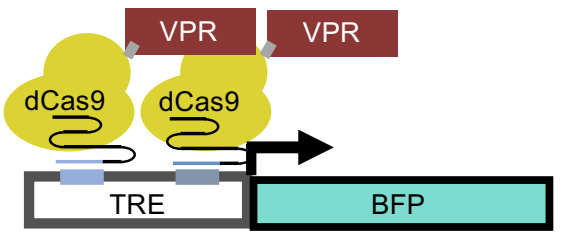

C
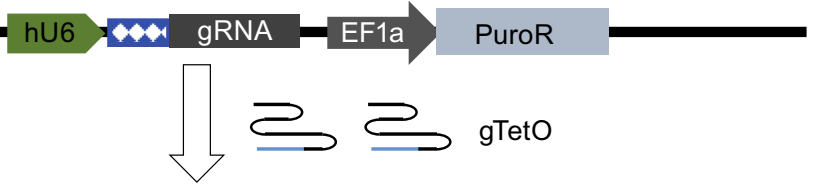

\section{PR}

293FT cells
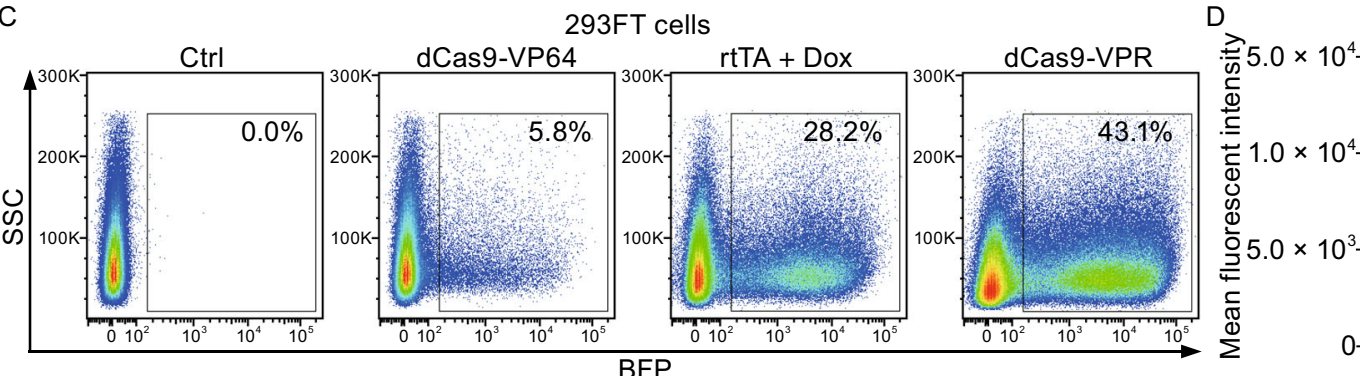

BFP

$E$

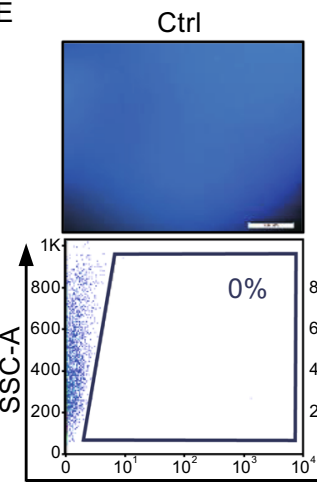

H9 hESC

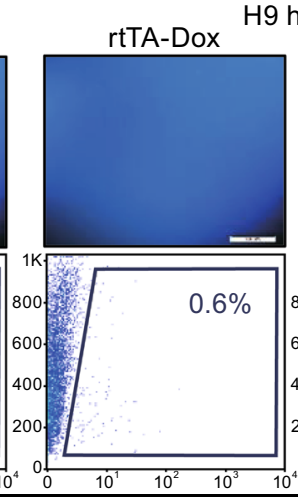

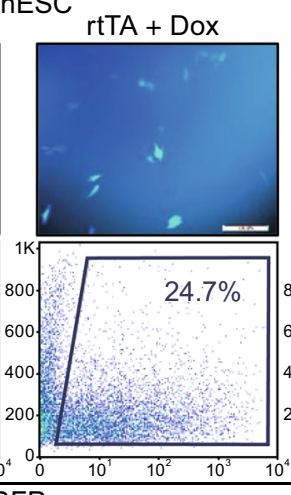

BFP
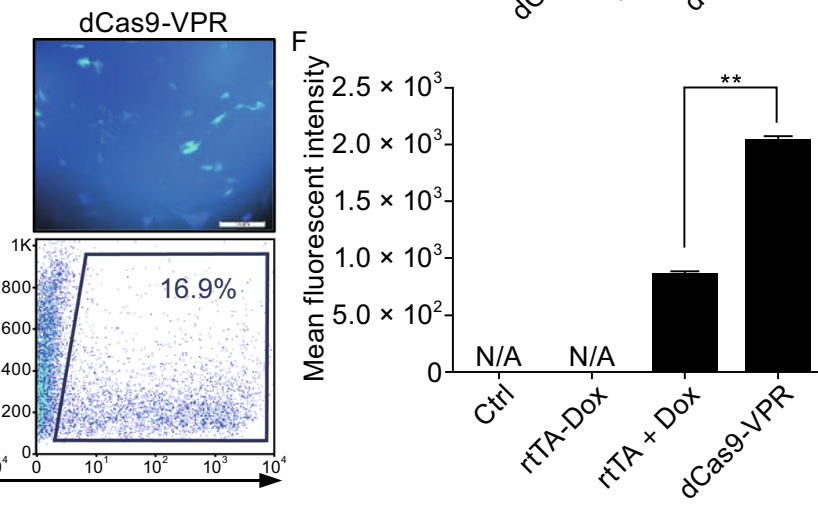

Figure 1. The dCas9-VPR system leads to robust transcription activation in human cell lines. (A) Schematic diagram of the gRNA guided dCas9-VPR gene activation system that consists of two parts: one plasmid contains dCas9-VPR driven by a CAG promoter; another plasmid contains gRNA targeting the promoter of the gene of interest driven by the human U6 promoter, in this case gTetO, and a PuroR selection cassette driven by an EF1a promoter. Upon co-transfection of the two plasmids, dCas9-VPR can activate the BFP transcription downstream of the TRE promoter. (B) Tet-On system: rtTA protein can bind to the TRE promoter and drive expression of the down-stream BFP gene in the presence of Dox. (C) 293FT cells were transfected with the reporter plasmid containing BFP driven by the TRE promoter. They were either co-transfected with dCas9-VPR or dCas9-VP64 and gTetO plasmids, or with the CAG-rtTA plasmid. Dox was added immediately after transfection. Cells were harvested 2 days after transfection and the fluorescence was analyzed using flow cytometry. (D) Bar graph quantification of mean fluorescent intensity analyzed using the FlowJo software v7.6.1. ${ }^{* \star *} P<0.001,{ }^{* \star \star \star} P<0.0001, n=3$. (E) H9 hESCs were electroporated with either rtTA or dCas9-VPR + gTetO plasmids together with the TRE-BFP plasmid. Dox was added immediately after electroporation. Cells were harvested 3 days after electroporation and analyzed using flow cytometry. $(F)$ Bar graph quantification of the mean fluorescent intensity analyzed using the FlowJo software v7.6.1. N/A, not applicable. ${ }^{* *} P<0.01, n=2$. 
A

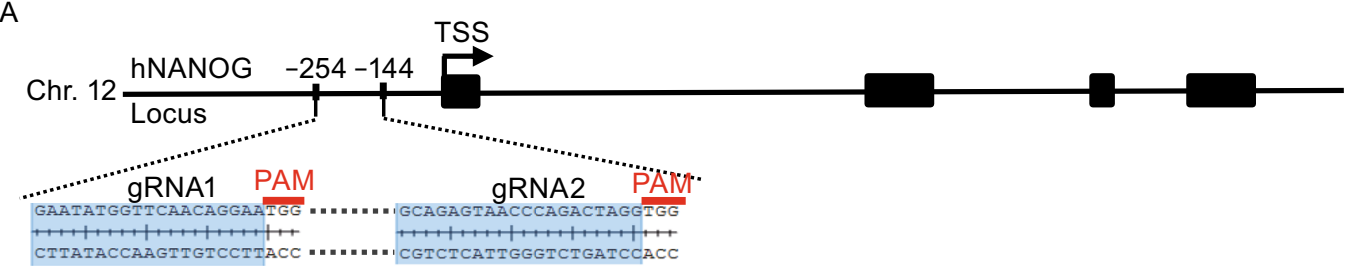

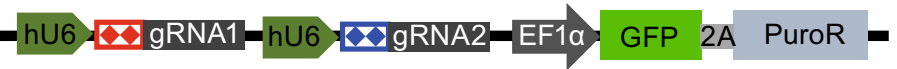

$$
己 \sqsupseteq \text { gNANOG }
$$

\section{VPR $\quad$ VPR}

B
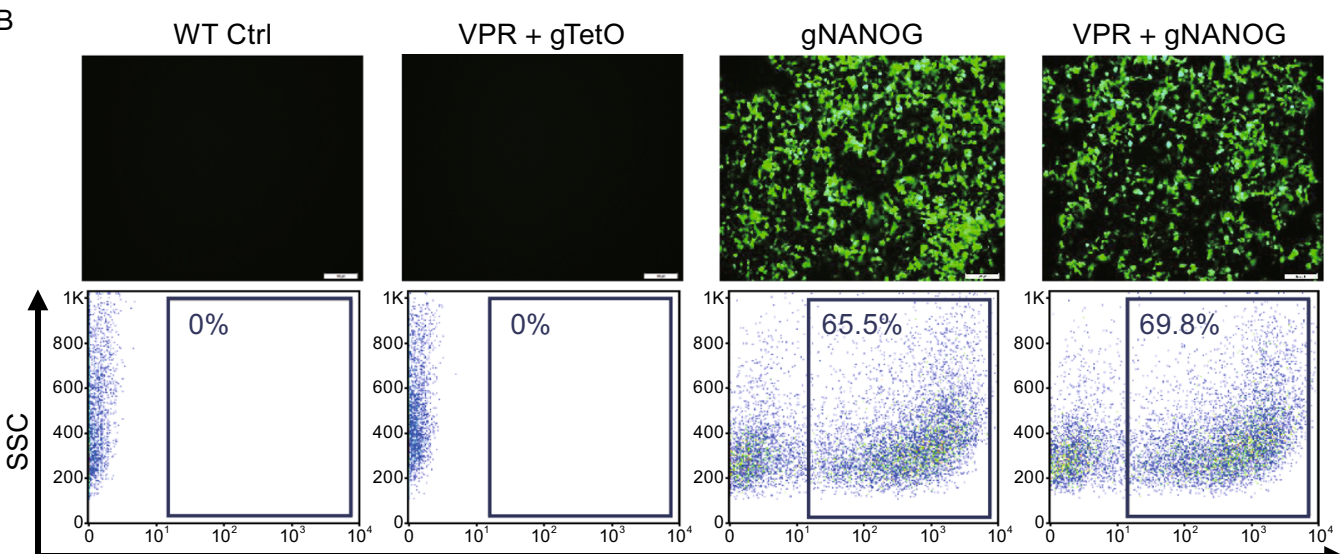

dCas9 dCas9

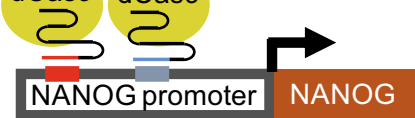

D
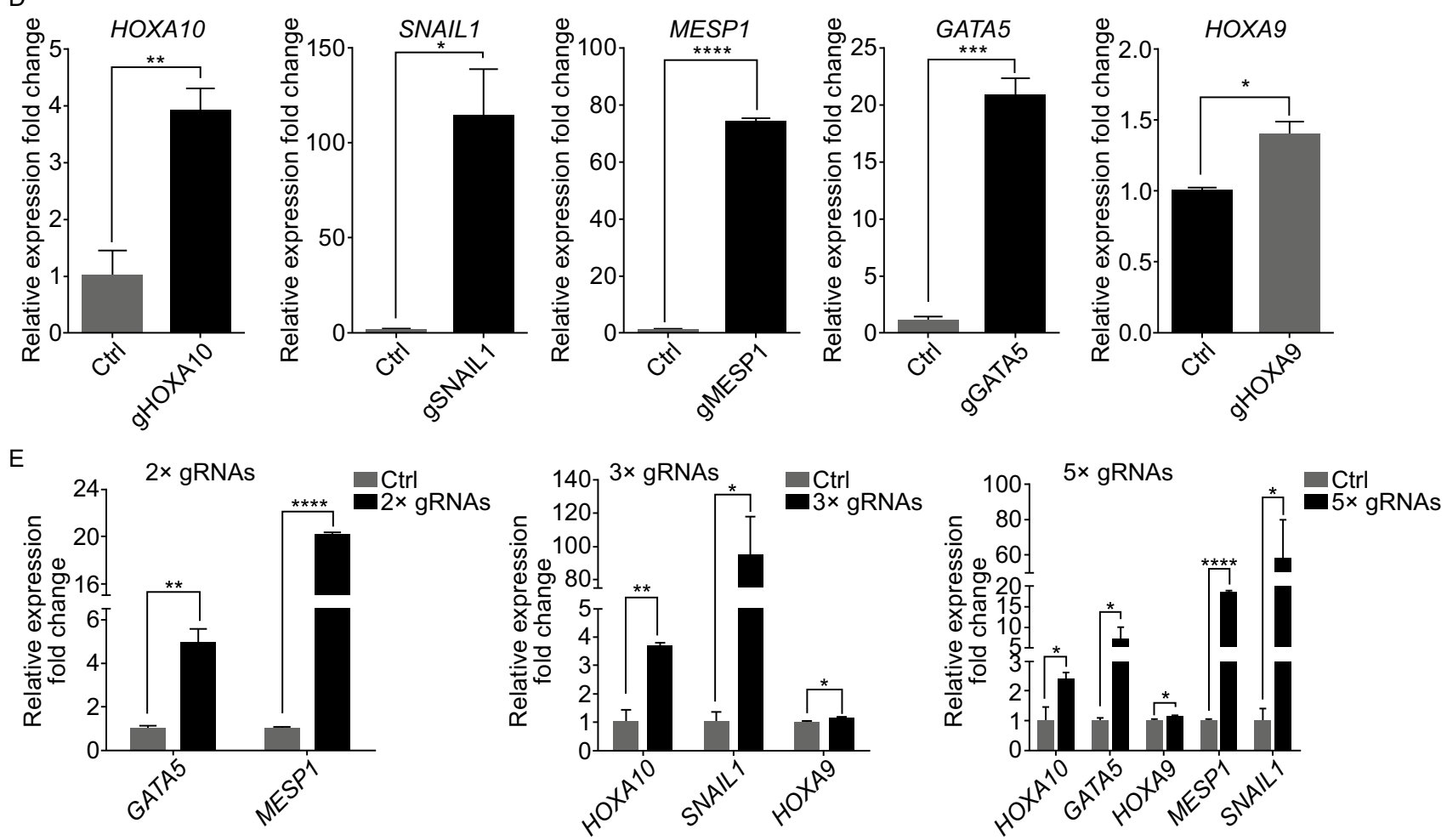
4 Figure 2. DCas9-VPR can be used to activate single or multiple genes in 293FT cells. (A) NANOG gRNA targeting sites were located at $-254 \mathrm{bp}$ and $-144 \mathrm{bp}$ upstream of the NANOG transcription starting site (TSS); protospacer-adjacent motif (PAM) sequences in red; black boxes indicate exons. (B) DCas9-VPR and gNANOG plasmids were co-transfected into 293FT cells. DCas9-VPR and gTetO plasmids were used as control. Top panels, fluorescence images of transfected cells; gNANOG plasmid transfected cells showed strong GFP fluorescence. Bottom panel, flow cytometry analysis of $\mathrm{GFP}^{+}$cells in each group. (C) Q-PCR analysis of NANOG expression 2 days after transfection; the dCas9-VPR system showed nearly 150-fold up-regulation of NANOG mRNA. Relative gene expression values were normalized against GAPDH. Error bars represent SEM. ${ }^{* *} P<0.01, n=3$. (D) Activation of endogenous genes by dCas9-VPR. DCas9-VPR was co-transfected with gRNA pairs targeting HOXA10, SNAIL1, MESP1, GATA5 or HOXA9, respectively. Cells were harvested 2 days after transfection and subjected to Q-PCR analysis. All tested genes showed significant upregulation compared to the control group. All expression levels were normalized against GAPDH. Error bars represent SEM. ${ }^{\star} P<0.05,{ }^{\star \star} P<0.01,{ }^{* \star *} P<0.001$, ${ }^{\star * \star \star} P<$ $0.0001, n=3$. (E) Simultaneously activation of multiple endogenous genes in 293FT cells. DCas9-VPR was co-transfected with $2 \times$ gRNAs (gMESP1, gGATA5), 3× gRNAs (gHOXA10, gSNAIL1, gHOXA9) or 5× gRNAs (gHOXA10, gSNAIL1, gMESP1, gGATA5 and gHOXA9). Cells were harvested 2 days after transfection. Q-PCR analysis confirmed coupregulation of multiple genes targeted by pooled gRNAs. All expression levels normalized against GAPDH. Error bars represent SEM. ${ }^{\star} P<0.05,{ }^{* \star} P<0.01,{ }^{* \star * *} P<0.0001, n=3$.

red) can be detected in colonies with GFP fluorescence. Upon Dox induction, stronger NANOG was also visible in Tet-On system transfected cells and co-localized with the H2B-mCherry (Fig. 3A). Quantitative PCR (Q-PCR) and Western blot confirmed the elevated NANOG level induced by either dCas9-VPR + gNANOG or NANOG CDS. The transcript level of another pluripotency marker gene, OCT4, was increased synergistically (Fig. 3B). Western blot analysis confirmed the upregulation of NANOG and OCT4 proteins in transiently transfected $\mathrm{H} 9$ cells (Fig. 3C). We generated a transgenic hESC line constitutively expressing dCas9-VPR and observed no cytotoxicity, decrease in pluripotency gene expression, or change in cell morphology for long-term cultures (Fig. 3D and 3E). This suggests that the dCas9-VPR system is suitable for gene activation studies in hPSCs.

\section{Generation of an inducible idCas9-VPR hESC knock-in line}

To achieve efficient, tunable, and reversible gene activation while avoiding compromising the genome integrity of hPSCs, we engineered an iVPR system by inserting the CAG promoter driving the rtTA expression cassette and the TRE promoter driving the dCas9-VPR cassette into the two alleles of the AAVS1 locus on chromosome 19. H9 hESCs were cotransfected with two donor plasmids containing dCas9-VPR and M2rtTA, as well as a pair of Cas9 nickase plasmids with AAVS1 targeting gRNAs to induce DNA double-strand break (DSB) and homology recombination (HR) (Fig. S2A). After puromycin and neomycin double selection for 2 weeks, we picked and expanded 17 clones. Upon addition of Dox, all the clones showed clear induction of dCas9-VPR protein expression (Fig. S2B). Genomic DNA PCR was performed to select correct targeted clones and rule out random insertions (Fig. S2C). Clone 2, 6 and 8 had targeted insertion at both AAVS1 alleles and without any random insertion (Fig. S2C). They were chosen for further analysis. Southern blot confirmed that in all three clones, both alleles of AAVS1 contained the correct insertion (Fig. 4A and 4B). Q-PCR analysis showed that in hESCs, without Dox treatment, little dCas9VPR transcript could be detected, while after Dox addition, strong dCas9-VPR expression was induced (Fig. 4C). Karyotype analysis showed that all three clones had normal 46XX karyotype (Fig. S2D). IVPR clone 2 was chosen for further study. Without Dox, we could not detect any dCas9VPR protein in iVPR cells. The dCas9-VPR protein appeared after $12 \mathrm{~h}$ of Dox addition and reached a plateau at $24 \mathrm{~h}$ (Fig. 4D). While $6 \mathrm{~h}$ after Dox withdrawal, the dCas9VPR protein decreased, by $12 \mathrm{~h}$, it decreased to a low level and could not be detected anymore after $24 \mathrm{~h}$ (Fig. 4D). The induction of dCas9-VPR from the AAVS1 locus was not affected by differentiation. We induced mesoderm differentiation by culturing cells in an RPMI medium supplemented with albumin, ascorbic acid, transferrin, selenite, BMP4 (5 ng/mL) and CHIR99021 (2 $\mathrm{mmol} / \mathrm{L})$ as described by Burridge et al. (2015). Q-PCR analysis showed that after 3 days of differentiation, pluripotency marker genes OCT4 and SOX2 were significantly downregulated, while dCas9-VPR was highly expressed as long as Dox was present, regardless whether cells were in hESC culture medium E8 or in the differentiation medium (Fig. 4E). Genes related to mesoderm differentiation and epithelial to mesenchymal transition, such as SNAIL, were strongly upregulated by BMP4 and CHIR99021, confirming that hESCs had taken a mesoderm fate (Fig. 4E). These results suggest that the iVPR hESC line can be used for efficient and reversible gene activation.

\section{Upregulation of NANOG by dCas9-VPR promoted naïve state of pluripotency}

The iVPR system provided a unique platform to investigate gene functions through activation from the endogenous locus. NANOG is a key regulator of pluripotency. We generated iNANOG hESCs by transfecting the PiggyBac based gNANOG plasmid described earlier into iVPR clone 2, 6, and 8 , followed by FACS selection of $\mathrm{GFP}^{+}$cells. Q-PCR analysis showed that after 2 days of Dox treatment, only 
A
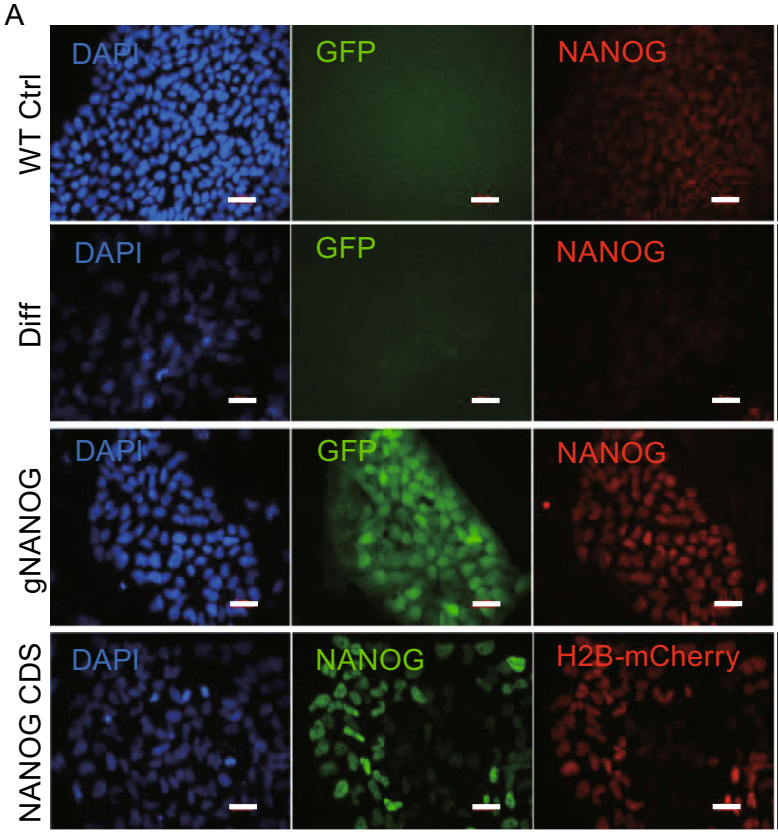

$\mathrm{D}$
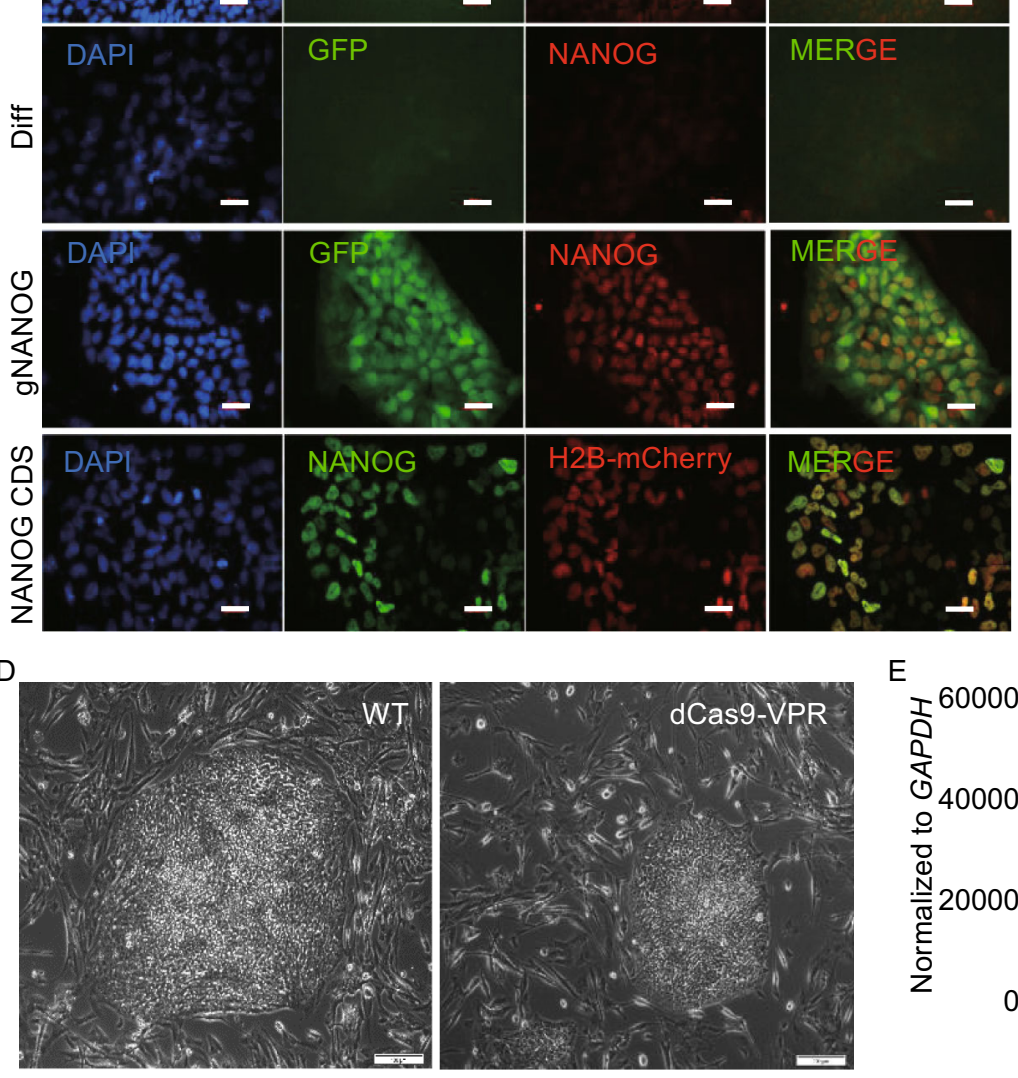
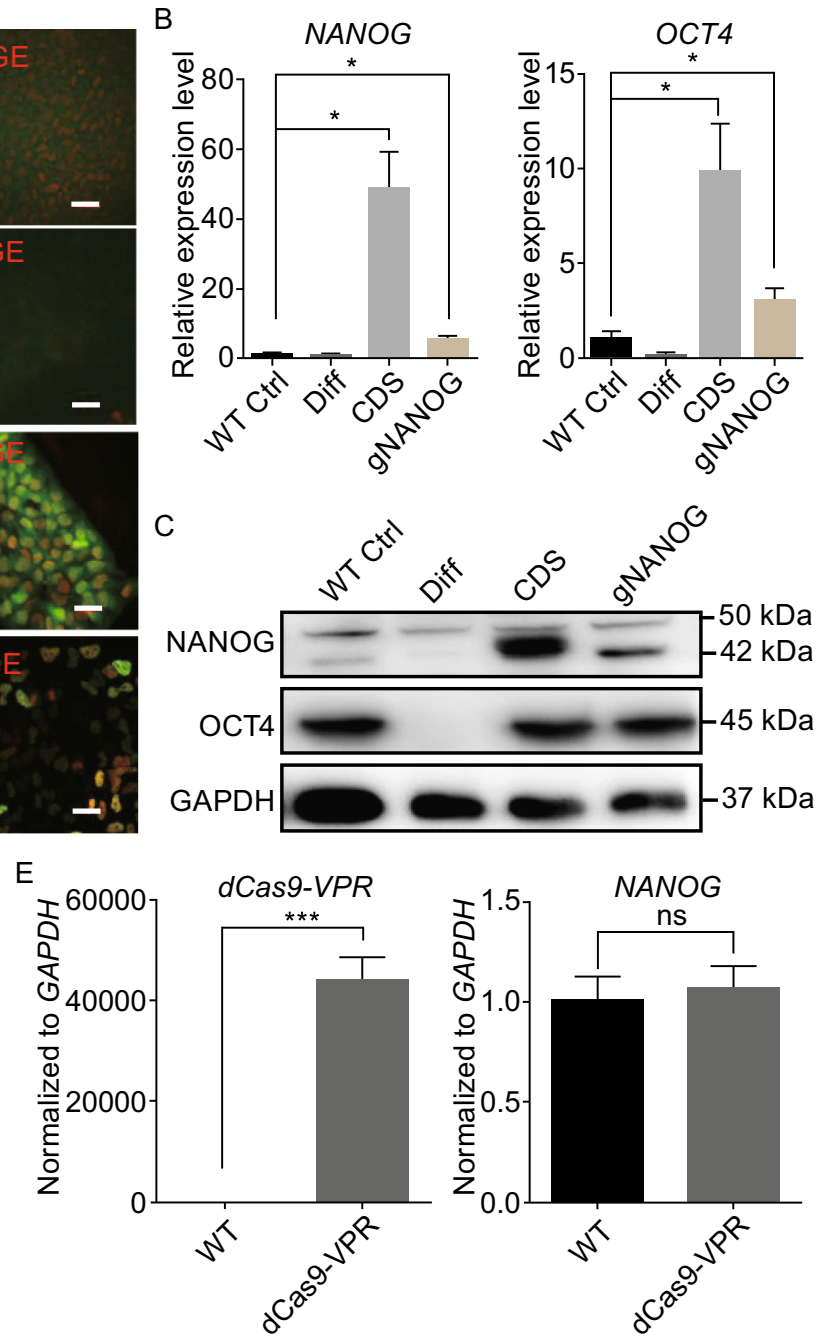

Figure 3. Activation of endogenous NANOG gene in hESCs by dCas9-VPR. (A) Immunostaining showing upregulation of NANOG protein by the dCas9-VPR system. Cells were fixed 5 days after transfection. WT Ctrl, untransfected H9 cells; Diff, differentiated $\mathrm{H} 9$ cells induced by $10 \mu \mathrm{mol} / \mathrm{L}$ retinoic acid (RA); gNANOG, H9 cells co-transfected with dCas9-VPR and gNANOG plasmids; NANOG CDS, cells co-transfected with CAG-rtTA and TRE driving NANOG-2A-H2B-mCherry. Dox were added immediately after electroporation. All plasmids were based on the PiggyBac system and co-transfected with a plasmid containing HyperPB transposase driven by a CAG promoter. Scale bar, $20 \mu \mathrm{m}$. (B) Q-PCR analysis of NANOG and OCT4 expression in H9 cells 5 days after transfection. All expression levels normalized against GAPDH. Error bars represent SEM. ${ }^{*} P<0.05, n=3$. (C) Western blot analysis of NANOG and OCT4 protein expression in H9 hESCs. Cells were harvested 5 days after transfection without selection.

(D) DCas9-VPR constitutive expressing H9 cells showed similar clone morphology after long-term culture. Scale bar, $100 \mu \mathrm{m}$.

(E) Q-PCR result showing dCas9-VPR constitutive expressing H9 cells and wild-type H9 cells expressed similar amount of NANOG.

All expression levels normalized against GAPDH. Error bars represent SEM. ns. $P>0.05,{ }^{* * *} P<0.001, n=3$.

iNANOG cells showed a significant increase (about 18 folds) in the NANOG mRNA level, while iVPR cells, with or without Dox, or iNANOG cells without Dox did not show any change in NANOG expression, indicating that the iNANOG system is tightly regulated (Fig. 5A). We also tested the time window of NANOG down-regulation after Dox withdrawal. NANOG mRNA was unchanged during the first $12 \mathrm{~h}$ and decreased after $24 \mathrm{~h}$. It approached the background level after $48 \mathrm{~h}$
(Fig. 5B). We next examined the change in NANOG protein level after Dox addition and withdrawal. Western blot revealed that dCas9-VPR protein became detectable $12 \mathrm{~h}$ after Dox induction and reached a significant level after $24 \mathrm{~h}$ (Fig. 5C, dCas9, long exposure; LE). Accordingly, NANOG protein showed an obvious increase after $24 \mathrm{~h}$ and maintained at high level as long as dCas9-VPR was present (Fig. 5C, NANOG, LE). On the other hand, $6 \mathrm{~h}$ after Dox 
A

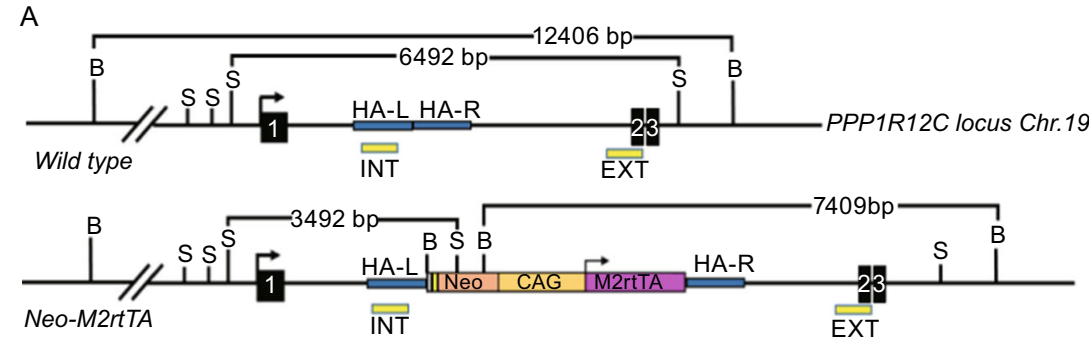

B

$5^{\prime}$ internal probe

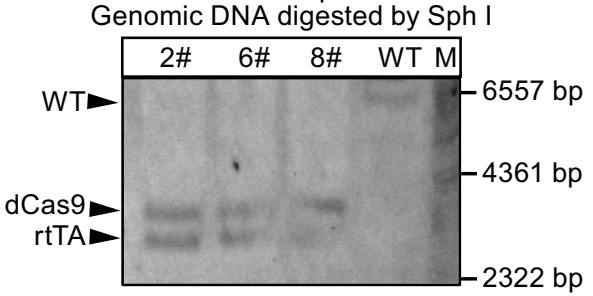

3 ' external probe Genomic DNA digested by BgI II
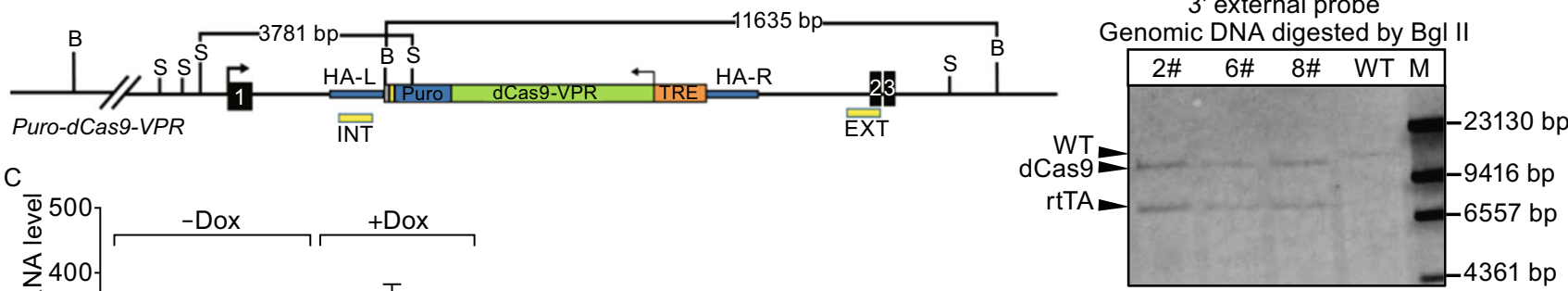

$\mathrm{C}$
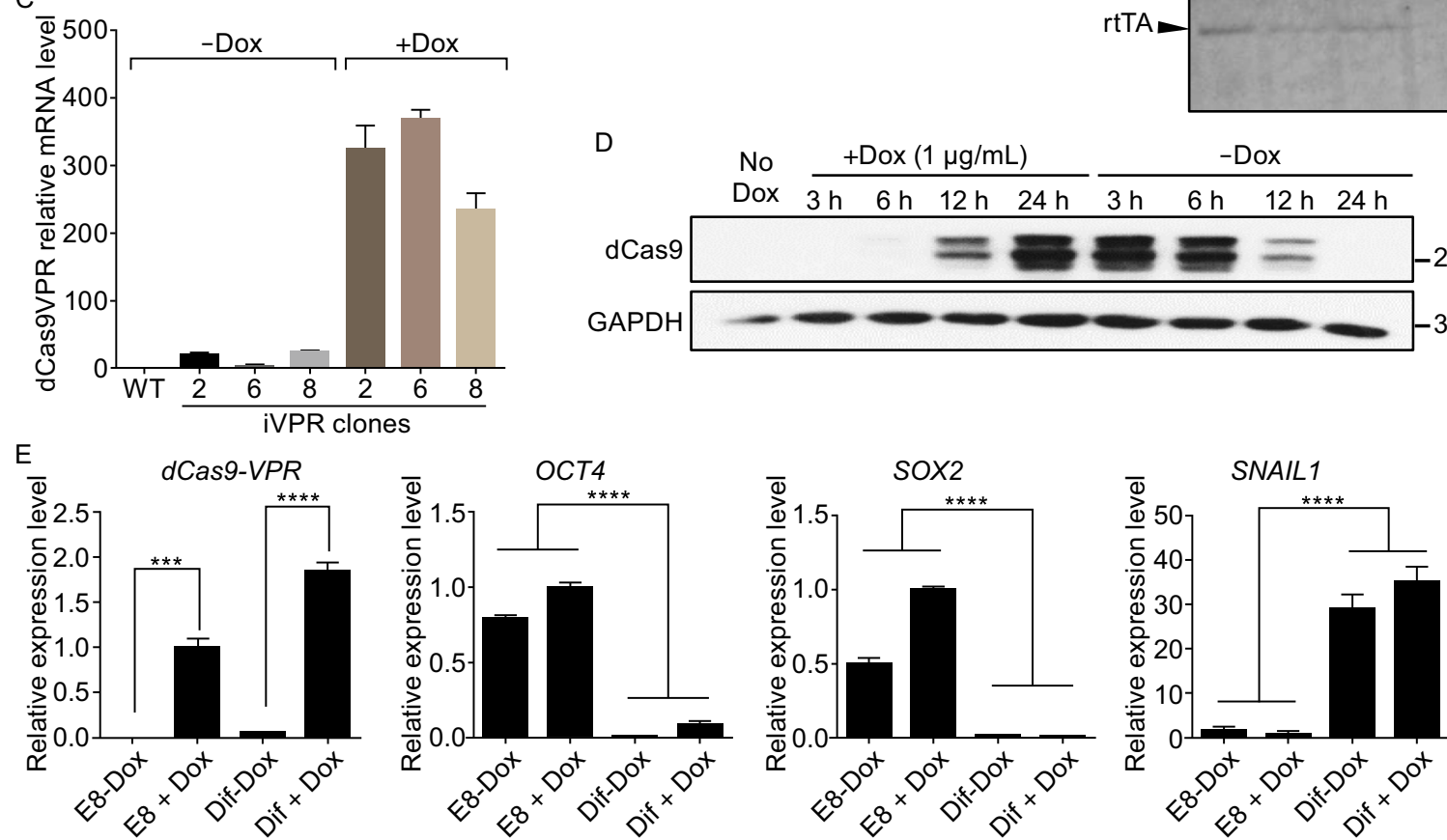

Figure 4. Generation of the iVPR hESC line. (A) Schematic view of wild type, targeted AAVS1 locus, and positions of Southern blot probes. B (Bgl II site), S (Sph I site), EXT (external probe), INT (internal probe). The sizes of the expected bands are indicated at the top. Blue lines indicate homology to the PPP1R12C intron. HA-L and HA-R, left and right homology arms. (B) Southern blot confirmed the correct targeted AAVS1 locus in the iVPR clone 2\#, 6\#, 8\#. M, marker. (C) Q-PCR analysis of dCas9-VPR transcript levels with or without Dox treatment. Expression levels were normalized against GAPDH. Error bar represents SEM. (D) Western blot of dCas9VPR protein level upon Dox addition and after Dox withdrawal in idCas9-VPR clone 2. The time points are indicated at the top. (E) Q-PCR showing that the induction of dCas9-VPR was not affected by differentiation. Cells were induced to undergo mesoderm differentiation for 3 days in the presence or absence of Dox. Gene expression levels were all normalized against GAPDH. Error bar indicates SEM. ${ }^{* \star *} P<0.001,{ }^{* \star *} P<0.0001, n=3$.

removal, the dCas9-VPR protein decreased significantly (Fig. 5D, dCas9, short exposure; SE). The decline of the dCas9-VPR protein was most apparent during the first $24 \mathrm{~h}$. After 4 days without Dox, dCas9-VPR protein became almost undetectable (Fig. 5D). Similarly, the NANOG protein level dropped to the background level after 4 days of Dox withdrawal (Fig. 5D). Q-PCR analysis showed that after Dox induction, iNANOG significantly upregulated naïve state related genes such as OCT4, PRDM14, GDF3, and LEF$T Y B$, while the early differentiation genes such as AFP was significantly downregulated (Fig. 5E). XIST, a long noncoding RNA involved in $X$ chromosome inactivation were also downregulated after NANOG induction (Fig. 5F). The expression of SSEA3, a more rigorous pluripotency cell 
A
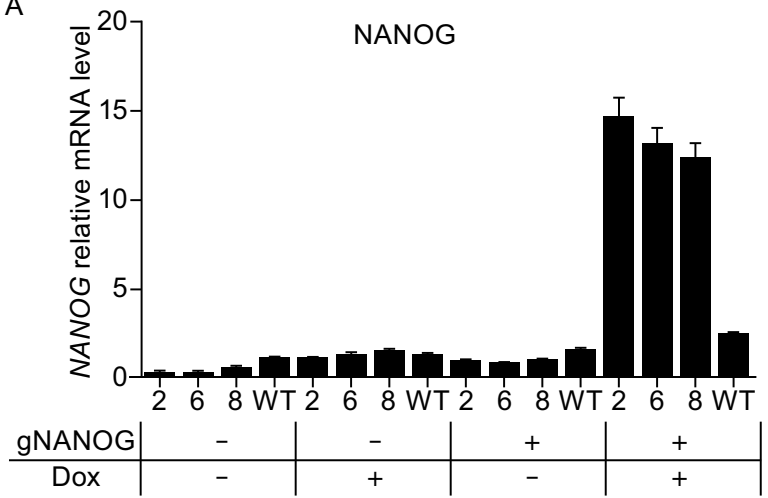

C

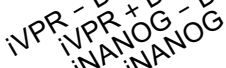

$\mathrm{F}$

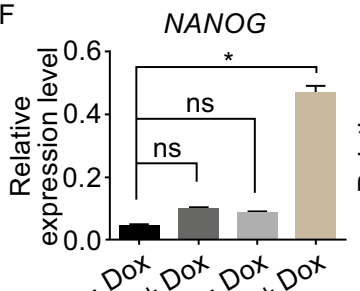

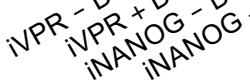

$\mathrm{H}$

-Dox

+Dox
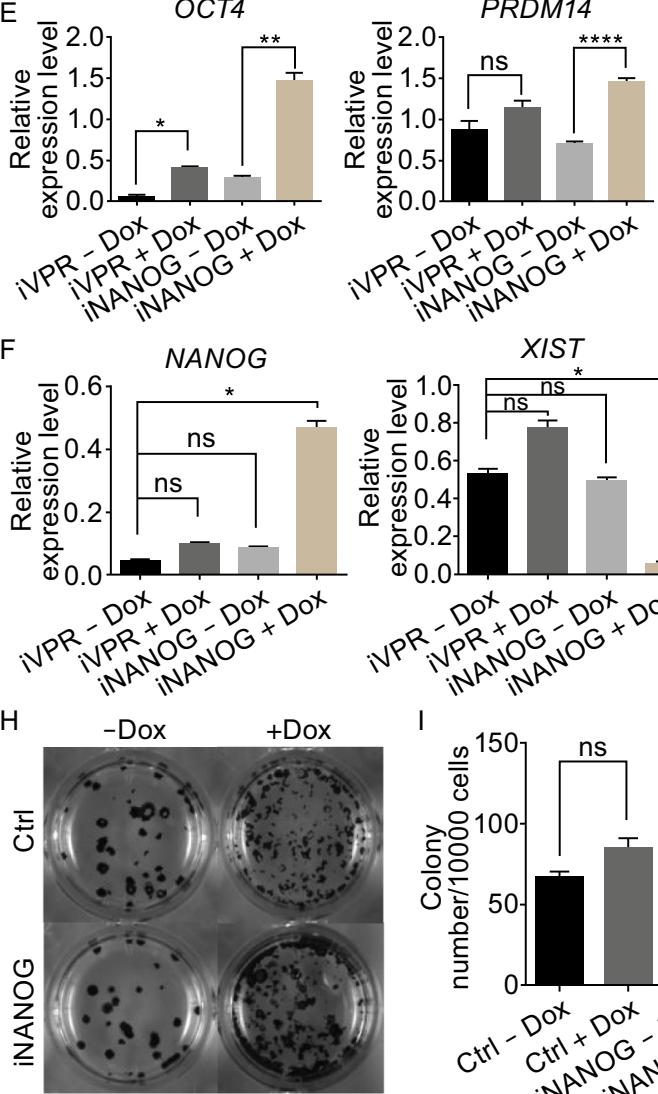

ivi intina

$X I S T$
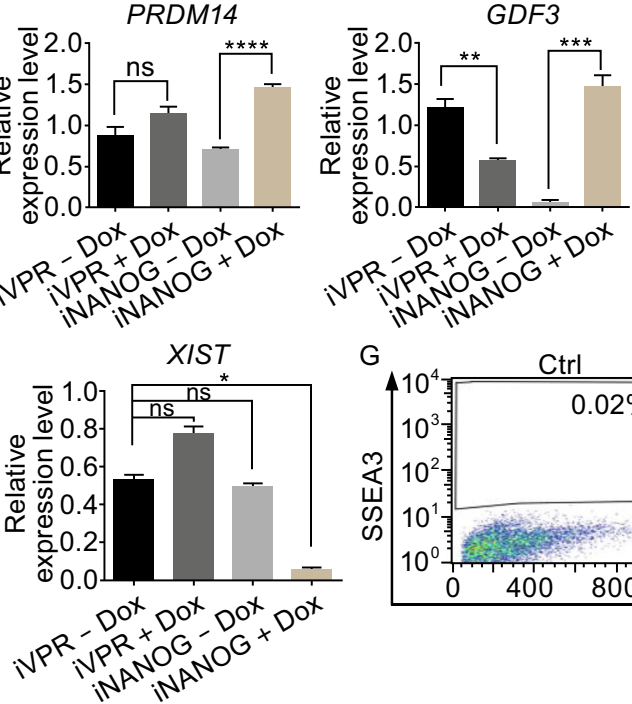

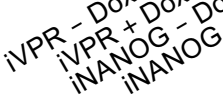

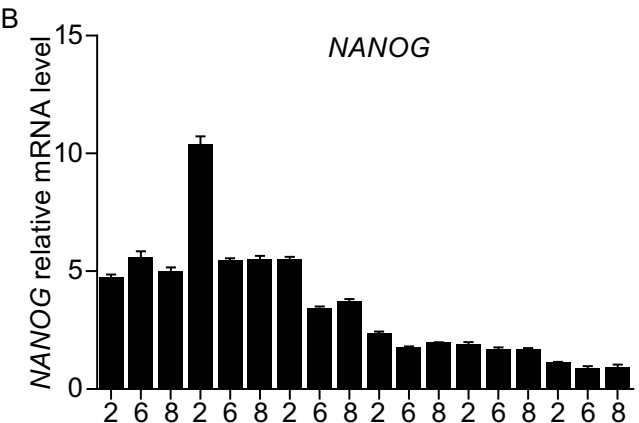

$\begin{gathered}\text { Dox } \\ \text { withdraw }\end{gathered}+|12 \mathrm{~h}| 24 \mathrm{~h}|48 \mathrm{~h}| 72 \mathrm{~h} \mid-$ D $\quad+$ Dox $\frac{-D o x}{-6 h-12 h-24 h-2 d-3 d-4 d-5 d-6 d-7 d}$ Dox

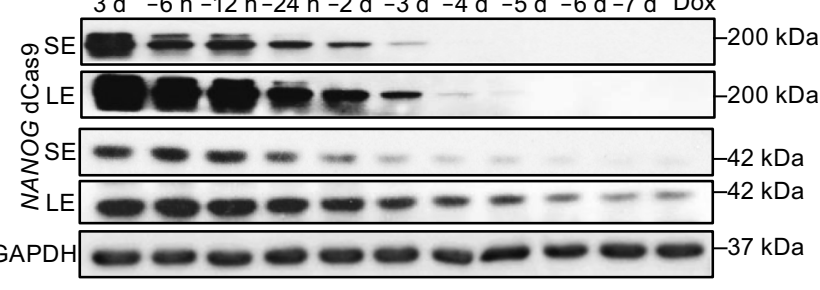

DF3
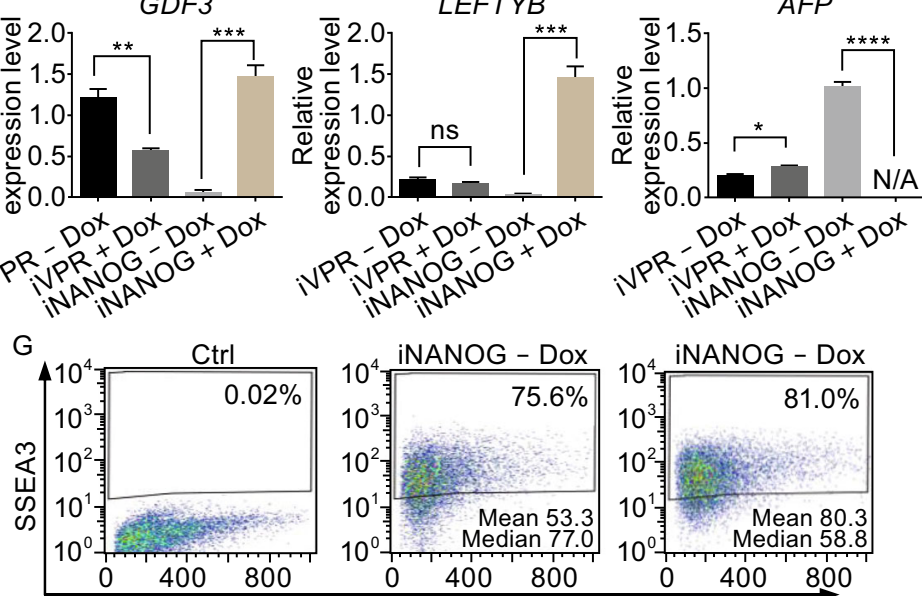

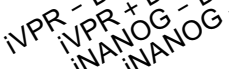

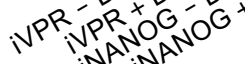

inainan
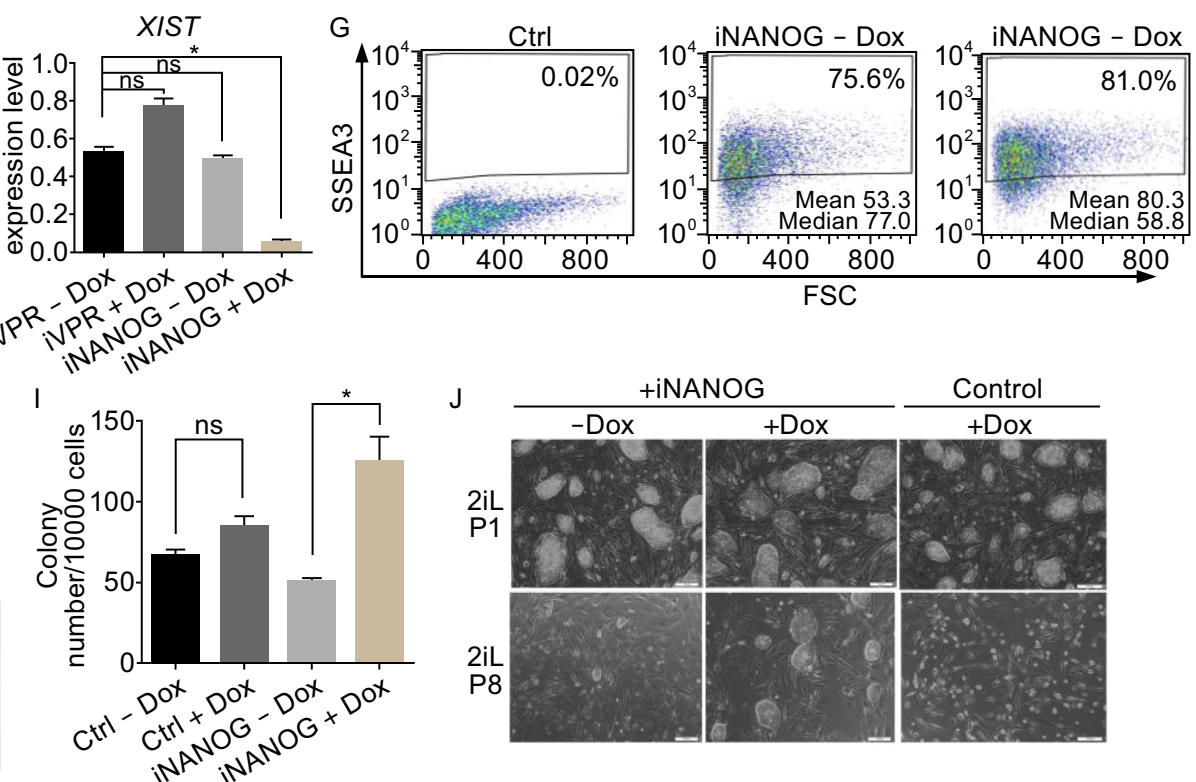

$\mathrm{K}_{\text {iNANOG cells w/o }}$

Dox in primed state

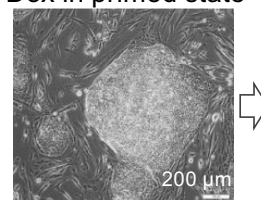

Dox induced iNANOG cells cultured in 2iL at P9
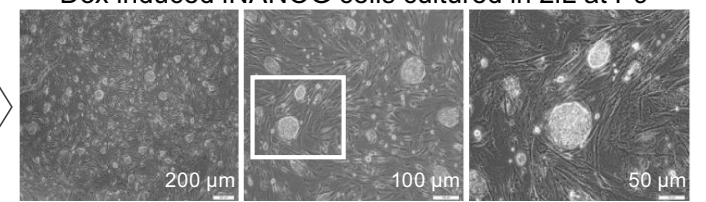
Figure 5. Upregulation of NANOG by dCas9-VPR promoted clonogenicity and the naïve state of pluripotency. (A) Q-PCR analysis of NANOG upregulation in iNANOG cells. IVPR clones 2, 6, and 8 were electroporated with gRNA expression plasmid targeting the NANOG promoter, as shown in Fig. 2A. GFP positive cells were purified by FACS and maintained as iNANOG cells. They were treated with or without Dox $(1 \mu \mathrm{g} / \mathrm{mL})$ for 2 days. NANOG expression level was normalized against $G A P D H$. Error bar represents SEM. (B) Q-PCR analysis of NANOG down-regulation in iNANOG cells. Dox was added for 2 days, then removed. Cells were harvested at different time points, as indicated. NANOG expression was normalized against GAPDH. Error bar represents SEM. (C) Western blot showing increased NANOG protein expression in iNANOG cells at different time points after Dox treatment. SE, short exposure; LE, long exposure; d, day; h, h. (D) Western blot showing NANOG protein expression decrease in iNANOG cells at different time points after Dox withdrawal. SE, short exposure; LE, long exposure; d, day; h, h. (E) Q-PCR analysis showing upregulation of pluripotency gene OCT4, PRDM14, GDF3, and LEFTYB, and down-regulation of differentiation gene AFP. Expression level all normalized against GAPDH. Error bar represents SEM. N/A, not applicable. ns. $P>0.05,{ }^{*} P<0.05,{ }^{* *} P<0.01,{ }^{* * *} P<0.001,{ }^{* * *} P<0.0001$, $n=3$. (F) Q-PCR analysis showing downregulation of XIST after $N A N O G$ induction. Expression level normalized against GAPDH. Error bar represents SEM. ns. $P>0.05$, ${ }^{*} P<0.05$, $n=3$. (G) Flow cytometry analysis showing increased SSEA3 expression after NANOG induction. Data analyzed using the FlowJo software v7.6.1. (H) Clonogenicity assay of iNANOG cells. Alkaline phosphatase assay (dark blue) was used to visualize undifferentiated colonies. (I) Bar graph quantification of the clonogenicity assay. ns. $P>0.05,{ }^{*} P<0.05, n=3$. (J) Morphology of iNANOG cells cultured in the 2iL medium. NANOG overexpression (iNANOG + Dox) promoted long-term cell growth in the $2 \mathrm{iL}$ medium. Representative images of passages 1 and 8 (P1 and P8) are shown. Scale bar, $100 \mu \mathrm{m}$. (K) Morphology of primed state iNANOG cells (without Dox) and Dox induced iNANOG cells (passage 9, P9) in the 2iL medium.

surface marker, was increased and became more homogeneous after NANOG elevation (Figs. $5 \mathrm{G}$ and S3). In addition to elevated expression of pluripotency genes, iNANOG cells also showed enhanced survival and proliferation abilities. Clonogenicity assay showed that after Dox induction, twice as many clones formed from dissociated iNANOG single cells (Fig. 5H and 5I). Finally, we tested whether NANOG upregulation by iVPR may facilitate hESCs to enter the naïve state of pluripotency. IVPR cells and iNANOG cells were cultured in 2iL medium which supplemented with ERK inhibitor PD0325901, GSK3 inhibitor CHIR99021, human LIF, and bFGF proteins with or without Dox addition (Silva et al., 2009; Takashima et al., 2014). Upon changing to the 2iL medium, hESCs colonies changed into a domed-shaped morphology and became more compact (Fig. 5J and 5K). INANOG cells without induction can only survive for no more than three passages in the 2iL medium (Fig. 5J). Interestingly, Dox induced iNANOG cells can grow in the 2iL medium for longer than 9 passages with single cell dissociation and a 1:15 passage ratio (Fig. $5 \mathrm{~J}$ and $5 \mathrm{~K}$ ). In contrast to iNANOG cells, Dox treated iVPR cells could not survive in 2iL conditions (Fig. 5J). Thus, upregulation of NANOG from its endogenous locus significantly improved single cell clonogenicity and permitted hESCs to grow in a naïve state culture environment.

\section{Upregulation of NANOG enabled hESCs to integrate with mouse ICM in vitro}

Entering the pluripotent ICM lineage is considered a more stringent test for naïve state ESCs (Gafni et al., 2013; Takashima et al., 2014). We next used in vitro human-mouse blastocyst chimera assay to assess the functionality of iNANOG cells (Fig. 6A). To exclude the influence of Dox treatment only, wild type hESCs stably carrying gNANOG (WTSG) were used as the control. For this series of experiments, we also added Forskolin (a cAMP agonist) into the 2iL medium, since it had been shown to promote hPSCs to enter the naïve state (Hanna et al., 2010; Ware et al., 2014; Duggal et al., 2015). We refer to this medium as 2iL/FK. iNANOG cells showed further enhanced proliferation in the 2iL/FK medium and were able to form large, dome-shaped colonies (Fig. 6B), while cells without NANOG overexpression could only form small colonies (Fig. S4A). E3.5 blastocysts were collected from ICR mice for hESC injection. iNANOG cells and WTSG cells cultured with or without Dox, in either the E8 or 2iL/FK medium, were dissociated into single cells. 10-15 single cells were injected into the blastocoel cavity and cultured in a 1:1 mixed KSOM:2iL/FK medium for $24 \mathrm{~h}$ (Fig. 6A). Because cells without NANOG overexpression only formed small colonies on feeder in the 2iL/FK medium, we could not obtain sufficient pure hESCs for blastocyst injection. Therefore, this group was omitted from this series of experiments. Since all cells used for injection contained GFP transgene expressed from the gNANOG plasmid, the location of human cells in the mouse blasocysts could be followed directly under the fluorescence microscope. 4-6 h after injection, most blastocysts contained GFP positive human cells (Fig. 6B and 6C). After $24 \mathrm{~h}$ of culture, many embryos still contained hESCs (Fig. 6B). We used time-lapse imaging to monitor the activity of hESCs in mouse blastocysts over time (Supplementary movie S1). Interestingly, endogenous NANOG overexpression strongly enhanced the survival of hESCs in mouse blastocysts. $12 \mathrm{~h}$ after injection, 2iL/FK cultured Dox induced gNANOG cells could be found in approximately $82 \%$ of blastocysts, while E8 cultured Dox induced gNANOG cells were alive in $73 \%$ of blastocysts (Figs. 6C and S4B). In contrast, without Dox induction, E8 cultured iNANOG cells could only be seen in $49 \%$ of injected blastocysts (Figs. 6 C and S4B). We next 

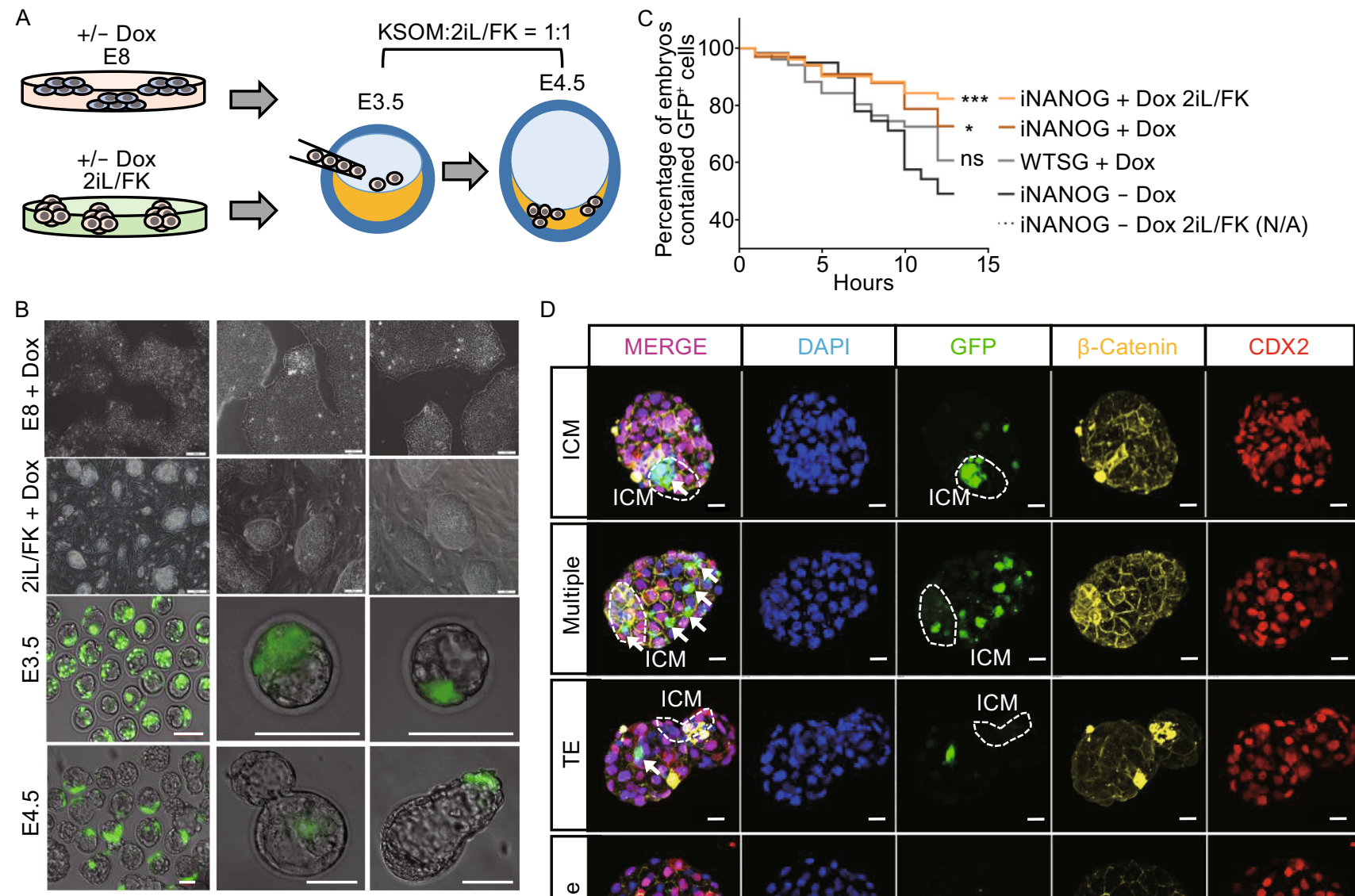

E
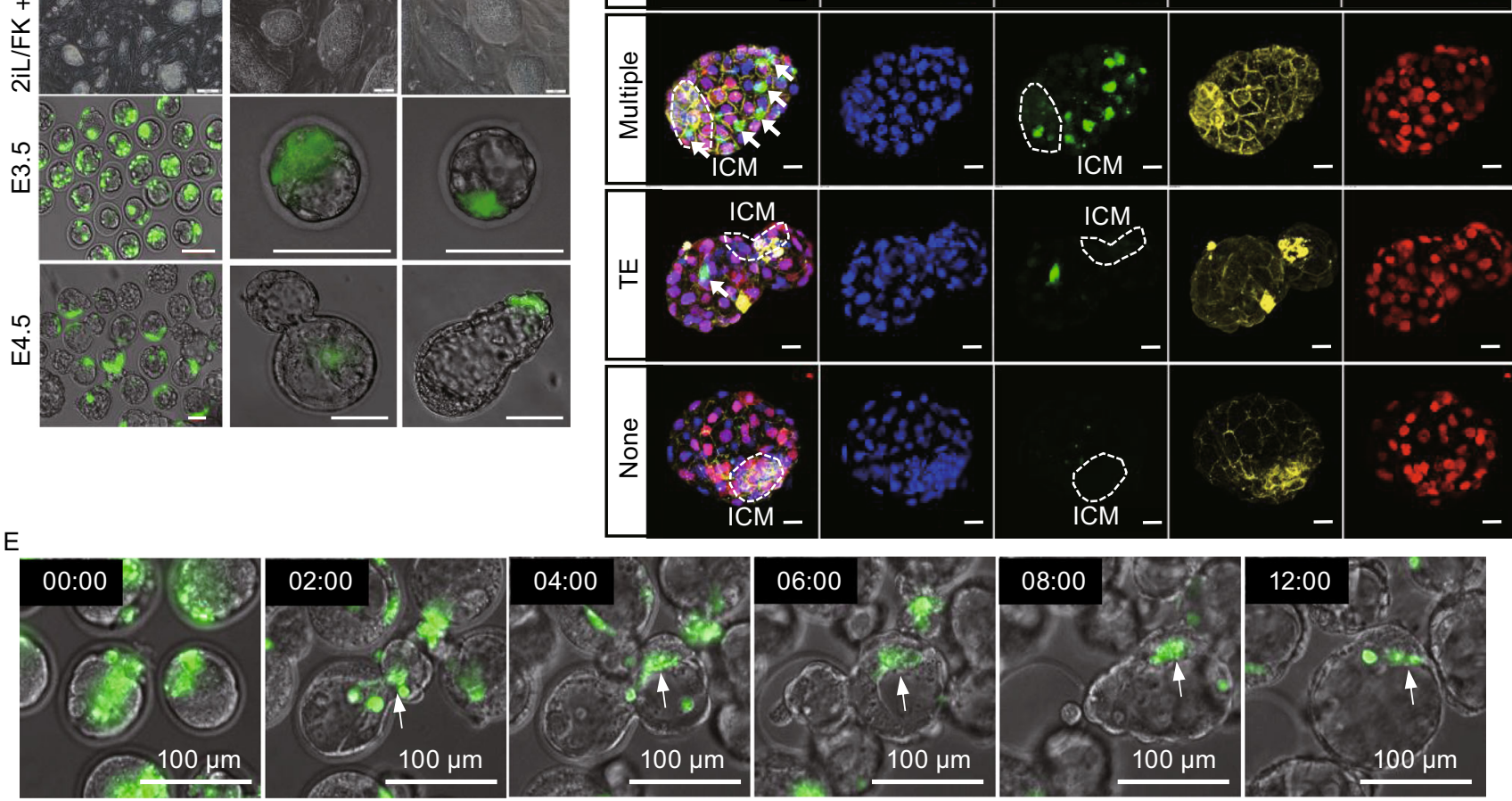

$\mathrm{F}$

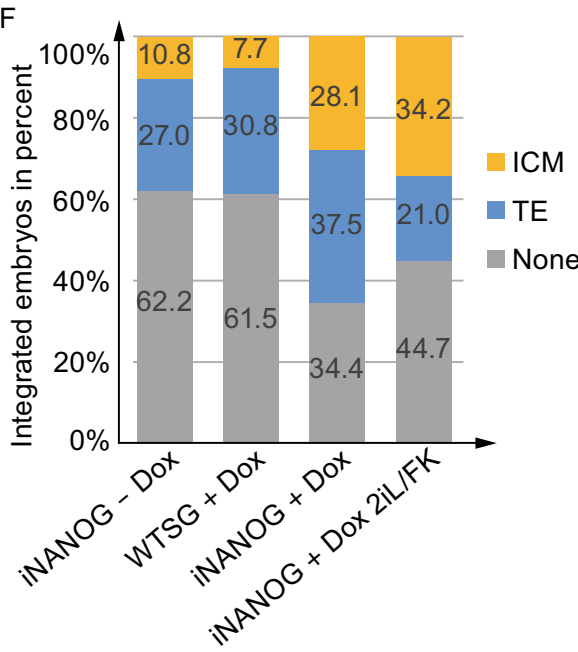

G

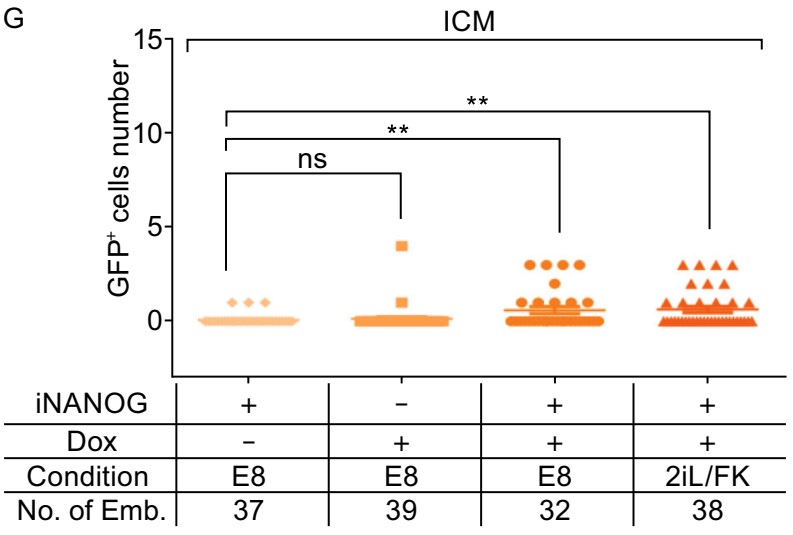


Figure 6. Upregulation of NANOG by idCas9-VPR promoted hESC survival and ICM integration in mouse blastocysts in vitro. (A) Cartoon showing in vitro hESC-mouse blastocyst chimera formation assay. INANOG cells were cultured in E8 or 2il/FK medium with or without Dox, then injected into E3.5 mouse blastocysts and cultured to E4.5 in KSOM: $2 \mathrm{il} / \mathrm{FK}=1: 1$ medium in vitro. (B) Morphology of iNANOG cells in culture and chimeric embryos. Top 2 rows, cells cultured in E8 or 2iL/FK on feeders; scale bar, $100 \mu \mathrm{m}$. Bottom 2 rows, E3.5 and E4.5 mouse blastocysts with iNANOG cells (GFP); scale bar, $100 \mu \mathrm{m}$. (C) Survival curve of hESC in mouse blastocysts over time. WTSG, wild type hESCs expressing NANOG gRNA. The $P$ value was calculated using the Log-rank (Mantel-Cox) test. ns. $P>0.05,{ }^{\star} P<0.05,{ }^{\star \star *} P<0.001$. Detailed information is provided in Fig. S4B. (D) Confocal images of E4.5 chimeric embryos. $\beta$-Catenin, yellow; CDX2, red; DNA, blue; iNANOG cells, green. The ICM region is highlighted by a dashed circle. Scale bar, $20 \mu \mathrm{m}$. (E) Selected frame from time-lapse movie of iNANOG-mouse blastocyst chimera. Arrows indicating iNANOG cells moved with mouse inner cell mass cells during blastocyst hatching. Scale bar, 100 $\mu \mathrm{m}$. (F) The proportion of blastocysts with hESC integration for E4.5 embryos. Blastocysts with GFP cells in both ICM and TE were counted as ICM. The percentage of embryos with ICM/TE/ None integration was labeled in the colored bar. (G) Dot graph showing the number of INANOG cells in the ICM region of E4.5 embryos. Cells, culture condition before injection, and number of embryos were as listed. Error bars represent SEM. ns. $P>0.05,{ }^{* *} P<0.01$.

analyzed the locations of the transplanted hESCs. Injected embryos were fixed after $24 \mathrm{~h}$ of culture, stained with CDX2 (a trophectoderm marker) and $\beta$-Catenin, and observed with a confocal microscope. Different integration patterns were shown: hPSCs integrated into the ICM region (ICM), in both the ICM and the trophectoderm (Multiple), only in the trophectoderm (TE), and disappeared (None) (Fig. 6D). We also performed live imaging to monitor the behavior of iNANOG cells in the mouse blastocyst. Interetingly, 2iL/FK cultured iNANOG cells tend to migrate with mouse inner cell mass cells as blastocyst hatching from the zona pellucida (Fig. 6E). NANOG overexpression significantly improved the percentage of cells remaining in blastocysts, and the 2iL/FK culture further increased the ICM integration proportion (Figs. 6F, 6G, and S4C). On average, two 2iL/FK or E8 cultured iNANOG cells could be found in the ICM region $24 \mathrm{~h}$ after injection, while without NANOG overexpression, hardly any GFP cells were seen in the ICM (Fig. 6G). Thus, upregulation of NANOG from its endogenous locus greatly enhanced cell survival and their subsequent ICM integration in hPSC-mouse blastocyst chimeras.

\section{DISCUSSION}

In this study, we generated an inducible CRISPR-ON hESC line by targeting the AAVS1 locus. Based on both our results and those of Chavez et al. (2015), dCas9-VPR appeared to be a stronger activator than VP64 to induce gene expression from both ectopic and endogenous promoters. It even led to a higher level of reporter gene activation compared with TetON rtTA, where VP64 was fused with Tet protein directly bound to the TRE elements. This is likely due to the combined effects of VP64, NF-KB transcactivating subunit p65, and the viral transcription factor Rta, which together can recruit a multitude of endogenous factors to achieve dramatically enhanced transcriptional activation. Other dCas9 based transcription activators have been generated. For example, Balboa et al. found increased activation ability with more VP16 fusing together. Using the longest version of dCas9-VP192 combined with inducible systems, they sucessfully facilitated human cell reprogramming and differentiation (Balboa et al., 2015). Konermann et al. engineered a structure-guided CRISPR synergistic activation mediator system (SAM), where they engineered gRNA2.0 by replacing the tetraloop and stem loop 2 of the original gRNA with a minimal hairpin aptamer that specifically binds to MS2 bacteriophage coat proteins (Konermann et al., 2014). By co-expression of dCas9-VP64, gRNA2.0, and MS2 fused with $\mathrm{p} 65$ and the activation domain of the human heat-shock factor 1 (HSF1), highly effective gene activation can be achieved (Konermann et al., 2014). Tanenbaum et al. constructed a SunTag system: dCas 9 was joined with 10 copies of GCN4 peptide (SunTag), while VP64 was fused with scFvGCN4 (the single-chain variable fragment (scFv) antibody of GCN4) (Tanenbaum et al., 2014). When co-expressed in the cell, SunTag was bound by ScFv-GCN4, and multiple copies of VP64 resulted activation of the target gene (Tanenbaum et al., 2014). Compared with the systems discussed above, which required introducing tandem repeat large cassette or the co-expression of two components in addition to the gRNA, dCas9-VPR is a simple and effective option.

In our study, we chose to insert the iVPR system into the AAVS1 locus, since it has been used as a 'safe habor' for transgene insertion in human stem cell systems (Dekelver et al., 2010). For example, Genga et al. constructed a GFP labled $\mathrm{H} 1 \mathrm{hESC}$ line by knocking-in a CAG-GFP into the AAVS1 locus. Besides, an inducible dCas9-KRAB gene inhibition system was also introduced into the GFP-H1 cells. By infecting sgRNA targeting the exdogenous CAG promoter, they successfully realized CRISPR based inhibition of exdogenous gene in hESCs (Genga et al., 2016). González et al. inserted the Dox inducible Cas9 system into the 2 alleles of the AAVS1 locus of HUES8 hESCs (González et al., 2014; Zhu et al., 2014; Zhu et al., 2015). The resulting iCRISPR hESC line enabled selection-free gene knock-out and the generation of lineage-specific knock-in reporters. This demonstrated that when Cas9 was expressed in a controllable manner from a suitable locus, the resulting cell line can be a powerful platform for genome editing in normally hard to transfect human stem cells (Zhu et al., 2015). Similarly, using the iVPR line, we found that the efficiency to generate an iNANOG line was much improved. Upon Dox addition and withdrawal, NANOG transcripts and proteins 
could be up- and down-regulated in a highly repeatable manner, which greatly facilitated downstream experiments. Recently, Ordovás et al. reported AAVS1-locus mediated transgene inhibition in hESCs, and that inhibition may due to different cassettes inserted into the locus (Ordovás et al., 2015). We tested the iVPR expression in both undifferentiated hESCs and after induction of mesoderm differentiation. The level of dCas9-VPR transcripts was even higher upon Dox treatment after mesoderm induction (Fig. 4E). The iVPR and iNANOG cells have been maintained for more than 6 months, and we did not observe any reduction in the level of $d$ Cas9-VPR or NANOG induced by Dox. Thus, results of us and other groups suggested that, in most cases, AAVS1 locus integration is a reliable approach to generate transgenic hPSCs.

NANOG is a master transcription factor for pluripotency in both human and mouse ESCs (Mitsui et al., 2003; Boyer et al., 2005; Chambers et al., 2007). During somatic cell reprogramming to pluripotent stem cells, ectopic expression of NANOG helped to speed up reprogramming and restrict partially reprogrammed cells to the ground state (Hanna et al., 2009; Silva et al., 2009). Different from mESCs, conventional cultured $\mathrm{hESCs}$ are in a primed state, similar to the epiblast stem cells in mice (Brons et al., 2007; Tesar et al., 2007). Recently, multiple groups reported methods to obtain naïve state hPSCs that resemble ground-state mESCs (Gafni et al., 2013; Duggal et al., 2015; Takashima et al., 2014; Theunissen et al., 2014). Takashima et al. showed that ectopic expression of NANOG and KLF2 could reset the selfrenewal requirements of hPSCs so that they can be grown in a medium containing ERK1/2 inhibitor PD0325901 and GSK3 inhibitor CHIR99021, and adopt a domed-shaped morphology similar to that of mESCs (Takashima et al., 2014). Here we increased the expression of endogenous NANOG by targeting a strong transcription activator, dCas9VPR, to its promoter. As expected, we observed upregulation of naïve state genes such as GDF3, PRDM14, and $\angle E F T Y B$ and downregulation of early differentiation gene $A F P$. Interestingly, these iNANOG cells showed a significantly improved survival ability and clonogenicity when cultured in the primed state, and they could grow in 2i plus LIF conditions for more than nine passages. The improved survival and self-renewal of iNANOG cells was not due to the effect of Dox treatment as described by Chang et al. (2014), because Dox treated iNANOG cells showed significantly higher clonogenicity over Dox treated iVPR cells (Fig. $5 \mathrm{H}$ and $5 \mathrm{I}$ ). The enhanced survival ability seemed to have a significant influence on whether hPSCs can integrate with the ICM of mouse blastocysts during in vitro culture. We found that even when iNANOG cells were in the primed state, after injection into mouse blastocysts, more cells remained inside the blastocysts and some of the cells were able to integrate with mouse ICM cells (Fig. 6F and 6G). Culturing iNANOG cells in 2iL/FK naïve state medium (Duggal et al., 2015) further improved the ICM integration rate (Fig. 6F and 6G). INANOG cells displayed highly dynamic interactions with mouse ICM cells, as observed in time-lapse movies (Supplementary movie S1). They migrated with mouse ICM cells as blastocysts hatched from zona pellucida. However, despite enhanced survival ability of iNANOG cells, many injected cells died over time. After $24 \mathrm{~h}$, more than $30 \%$ of injected blastocysts lost all iNANOG cells and more than $60 \%$ of blastocysts lost the injected hESCs if NANOG was not overexpressed (Fig. 6F). This was partially caused by poor survival of hESCs in the IVC- 1 and -2 media designed to culture peri-implantation mouse and human embryos (Bedzhov and Zernicka-Goetz, 2014; Deglincerti et al., 2016; Shahbazi et al., 2016) (Fig. S4D). Thus, to achieve better naïve hPSC and mouse ICM integration, a culture medium suitable for both mouse blastocysts and hPSCs may be needed. The effect of NANOG overexpression on cell survival and self-renewal is also in accordance with the observation that chromosome 12, where the NANOG gene is located, is the most frequently gained chromosome in culture adapted hPSCs (Baker et al., 2007) and during hiPSC generation (Taapken et al., 2011). Moreover, NANOG was reported to be upregulated by a number of factors such as STAT3, Hedgehog signaling, hypoxia, etc., in human cancers, and repression or ablation of NANOG inhibited tumor initiation (Gong et al., 2015). Thus, iNANOG hESCs, where the endogenous NANOG can be activated by dCas9-VPR in a controllable manner, may also be a good system to study the process of hPSC adaptation and cancerous transformation.

In summary, the iVPR hESC line generated and characterized in this study offered a convenient, stable, and highly controllable platform for gene activation studies. It can also be used to investigate the function of regulatory elements in the genome such as super enhancers as well as for genome wide screens using established human gRNA libraries.

\section{MATERIALS AND METHODS}

HESC culture

H9 hESCs (WiCell Institute) were maintained on inactivated mouse embryonic fibroblast (MEF) cells in standard hESC medium consisting of KO-DMEM (Invitrogen) supplemented with 1× Nonessential Amino Acids (NEAA) (Invitrogen), $0.1 \mathrm{mmol} / \mathrm{L}$ 2-mercaptoethanol (Sigma-Aldrich), $1 \mathrm{mmol} / \mathrm{L}$ GlutaMAX (Invitrogen), 20\% Knock-out serum-replacement (KOSR) (Invitrogen) and $4 \mathrm{ng} / \mathrm{mL}$ bFGF (Peprotech). Cells were cultured at $37^{\circ} \mathrm{C}$ in a humidified atmosphere with $5 \% \mathrm{CO}_{2}$ in air. They were passaged with $1 \mathrm{mg} / \mathrm{mL}$ collagenase IV (Invitrogen) and seeded onto MEFs. For feeder-free culture, hESCs were grown for more than three passages on Matrigel (growth factor reduced, BD Biosciences) in the absence of feeders in E8 medium (Invitrogen).

\section{Plasmid construction}

DCas9-VPR was constructed by fusing the nuclease deficient Cas9 (dCas9) with transcription activator VP64, p65, and Rta in tandem as described by Chavez et al. (Maeder et al., 2013). For constitutive 
expression, dCas9-VPR was placed behind a CAG promoter in a PiggyBac vector also containing a PGK promoter driving a hygromycin resistance gene. For inducible expression from the AAVS1 locus, dCas9-VPR was placed behind a TRE promoter in the AAVS1 homologous recombineering donor plasmid, as shown in Fig. S2A. DCas9-VP64 was constructed by fusing dCas9 with VP64. Tet-On system was obtained from Clontech (http://www.clontech.com). PiggyBac plasmids were generous gift from the Sanger institute, Cambridge, UK (http://www.sanger.ac.uk). The multiple NANOG gRNA expression plasmid was constructed by SynGene (http:// syngen.tech) as depicted in Fig. 2A.

\section{Naïve state culture condition for hPSCs}

For naïve state conversion, cells cultured in standard hESC medium on MEFs were dissociated to single cells using $0.05 \%$ trypsin/EDTA solution (Invitrogen), replated on MEFs, and cultured overnight in standard hESC medium supplement with $10 \mu \mathrm{mol} / \mathrm{L}$ Rho Kinase (ROCK)-inhibitor Y-27632 (Calbiochem). The next day, the standard medium was changed to the 2iL or 2iL/FK (for injection) medium, which consisted of KO-DMEM (Invitrogen), 20\% KOSR, 1×NEAA, $0.1 \mathrm{mmol} / \mathrm{L}$ 2-mercaptoethanol, $1 \mathrm{mmol} / \mathrm{L}$ GlutaMAX, $12 \mathrm{ng} / \mathrm{mL}$ bFGF, $10 \mathrm{ng} / \mathrm{mL}$ human recombinant LIF (Peprotech), $1 \mu \mathrm{mol} / \mathrm{L}$ ERK1/2 inhibitor PD0325901 (Peprotech), 3 mol/L GSK3 inhibitor CHIR99021 (Peprotech), $10 \mu \mathrm{mol} / \mathrm{L}$ Forskolin (Peprotech), and $50 \mu \mathrm{g} / \mathrm{mL}$ ascorbic acid (Sigma). HESCs changed to a dome-shaped morphology within 4-6 days after culturing in the 2iL or 2iL/FK medium and were passaged every 4 days as single cells using $0.05 \%$ trypsin/EDTA.

\section{Cardiac mesoderm differentiation from hESCs}

For cardiac mesoderm differentiation, hESCs maintained on Matrigel (growth factor reduced, BD Biosciences) in E8 were dissociated into single cells with Accutase (Invitrogen), then seeded onto Matrigelcoated tissue culture dishes at a density of $5 \times 10^{4}$ cells $/ \mathrm{cm}^{2}$ and cultured in E8 for 3 days. Then the medium was switched to the RPMI1640 medium supplemented with Albumin, Ascorbic acid, transferrin, selenite, $5 \mathrm{ng} / \mathrm{mL}$ BMP4 (R\&D Systems), and CHIR99021 to induce cardiac mesoderm formation.

\section{Quantitative PCR}

Total RNA was extracted with TRIZOL (Invitrogen). $1 \mu \mathrm{g}$ RNA of each sample was used for reverse transcription with Superscript III (Invitrogen). Q-PCR reactions were performed using GoTaq qPCR Master Mix (Promega) in a CFX96 Real-Time System (Bio-Rad). The relative expression level of each gene was normalized against the $\mathrm{Ct}$ (Critical Threshold) value of the house-keeping gene GAPDH using the Bio-Rad CFX Manager program. Primer sequences are listed in table S2.

\section{Antibodies, immunostaining, Western blot, and FACS analysis}

For immunostaining, cells were fixed in $4 \%$ paraformaldehyde (PFA) in PBS, permeabilized in $0.5 \%$ Triton X-100 (Sigma), blocked in 5\% normal goat serum (Origene) and incubated with primary antibodies against NANOG (1:200), SSEA3 $(1: 200)$ in $4^{\circ} \mathrm{C}$ overnight and detected by DyLight 488- or 549-conjugated secondary antibodies (Thermo). Nuclei were stained with DAPI (Sigma). A Nikon Ti-U fluorescence microscope was used for image acquisition. For western blot, cells were lysed in a RIPA buffer (Applygen, http:// applygen.com.cn) with Protease Inhibitor Cocktail (Roche). Total proteins were separated on a $12 \%$ SDS/PAGE gel, transferred to nitrocellulose membrane (Whatman). The membrane was blocked with $5 \%$ non-fat dry milk in TBST and then incubated with primary antibodies against Cas9 (Genetex, 1:1000), GAPDH (CWBio, 1:1000), OCT4 (Santa Cruz, 1:1000) and NANOG (Cell Signaling Technology, 1:1000). After washing, the membrane was incubated with anti-mouse or anti-rabbit peroxidase-conjugated secondary antibodies (ZSGB-Bio http://www.zsbio.com/). Bands recognized by antibodies were revealed by ECL reagent (Pierce). For FACS analysis, cells were first dissociated with $0.05 \%$ Trypsin in $0.2 \%$ EDTA and PBS. FACS was performed on a Fortessa flow cytometer (Becton Dickinson).

\section{Mouse blastocyst injection and in vitro culture}

The animal facility of Tsinghua University has been accredited by the AAALAC (Association for Assessment and Accreditation of Laboratory Animal Care International) and all animal protocols used in this study were approved by the IACUC (Institutional Animal Care and Use Committee) of Laboratory Animal Research Center of Tsinghua University. Mouse morula were collected from ICR females 2.5 days post-coitus and cultured in $\mathrm{KSOM}$ medium $(95 \mathrm{mmol} / \mathrm{L}$ $\mathrm{NaCl}, 2.5 \mathrm{mmol} / \mathrm{L} \mathrm{KCl}, 0.35 \mathrm{mmol} / \mathrm{L} \quad \mathrm{KH}_{2} \mathrm{PO}_{4}, 0.2 \mathrm{mmol} / \mathrm{L}$ $\mathrm{MgSO}_{4} \cdot 7 \mathrm{H}_{2} \mathrm{O}, 0.2 \mathrm{mmol} / \mathrm{L}$ glucose, $10 \mathrm{mmol} / \mathrm{L}$ sodium lactate, 25 $\mathrm{mmol} / \mathrm{L} \mathrm{NaHCO}_{3}, 0.2 \mathrm{mmol} / \mathrm{L}$ sodium pyruvate, $1.71 \mathrm{mmol} / \mathrm{L}$ $\mathrm{CaCl} \cdot 2 \mathrm{H}_{2} \mathrm{O}, 0.01 \mathrm{mmol} / \mathrm{L}$ EDTA, $1 \mathrm{mmol} / \mathrm{L}$ L-glutamine, $0.1 \mathrm{mmol} / \mathrm{L}$ EAA, $0.1 \mathrm{mmol} / \mathrm{L} \mathrm{NEAA}, 4 \mathrm{mg} / \mathrm{mL}$ BSA) at $37^{\circ} \mathrm{C}, 5 \% \mathrm{CO}_{2}$ for $24 \mathrm{~h}$ to get blastocysts (Hogan et al., 1986). HESCs were briefly treated with Accutase for single cell and injected $(\sim 10-15$ cells for each embryo) on a Nikon microscope fitted with piezo-driven Eppendorf NK2 micromanipulator, CellTram air and CellTram Vario. After injection, embryos containing hESCs were cultured in medium supplemented with naïve culture medium:KOSM (1:1) (Chen et al., 2015 ) in $37^{\circ} \mathrm{C}, 5 \% \mathrm{CO}_{2}$ incubator. After injected embryos reformed blastocoel, the chimera embryos were live cell imaged using Leica microscope fitted with a live cell imaging system and fixed after 24 $36 \mathrm{~h}$ post-injection for staining and confocal imaging. For embryo immunostaining, zona pellucida-free injected embryos were fixed with $3.5 \%$ paraformaldehyde, permeabilized in $0.5 \%$ Triton X-100 (Sigma) and blocked with 5\% BSA and then incubated with primary antibodies against CDX2 (BioGenex), $\beta$-Catenin (1:50, Abcam) and detected by DyLight 549- or 633- conjugated secondary antibodies (Thermo). Nuclei were stained with DAPI (Sigma). A Nikon-A1 fluorescence microscope was used for image acquisition.

\section{Statistical analysis}

Data are presented as mean \pm standard error of the mean (SEM). Statistical significance was determined by Student's $t$-test (two-tail) for two groups or one-way Analysis of Variance (ANOVA) for multiple groups using Graphpad software. $P<0.05$ was considered significant. 


\section{ACKNOWLEDGMENTS}

This work was supported by the National Basic Research Program (973 Program) (No. 2012CB966701), the National Natural Science Foundation of China (Grant No. 31171381 to J.N.), and core facilities of the Tsinghua-Peking University Center for Life Sciences. TNLIST Interdisciplinary research foundation grant 042003171 (to Z.X. and N.J.). We thank Dr. Danwei Huangfu for the AAVS1 homologous recombineering donor plasmids and $\mathrm{Dr}$. Xiaohua Shen lab for assistance in Southern blot.

\section{AUTHOR CONTRIBUTIONS}

J.G.: concept and design, collection and/or assembly of data, data analysis and interpretation, manuscript writing; D.M., R.H., M.Y., J. M.: collection and/or assembly of data; K.K. provided essential reagents, technical and scientific advice to the experiments and manuscript; Z.X.: concept and design; J.N.: concept and design, manuscript writing, and final approval of the manuscript.

\section{COMPLIANCE WITH ETHICAL GUIDELINES}

Jianying Guo, Dacheng Ma, Rujin Huang, Jia Ming, Min Ye, Kehkooi Kee, Zhen Xie, and Jie $\mathrm{Na}$ declare no conflict of interests.

This article does not contain any studies with human subjects performed by the any of the authors. All institutional and national guidelines for the care and use of laboratory animals were followed.

\section{OPEN ACCESS}

This article is distributed under the terms of the Creative Commons Attribution 4.0 International License (http://creativecommons.org/ licenses/by/4.0/), which permits unrestricted use, distribution, and reproduction in any medium, provided you give appropriate credit to the original author(s) and the source, provide a link to the Creative Commons license, and indicate if changes were made.

\section{REFERENCES}

Baker DEC, Harrison NJ, Maltby E, Smith K, Moore HD, Shaw PJ, Heath PR, Holden H, Andrews PW (2007) Adaptation to culture of human embryonic stem cells and oncogenesis in vivo. Nat Biotechnol 25:207-215

Balboa D, Weltner J, Eurola S, Trokovic R, Wartiovaara K, Otonkoski T (2015) Conditionally stabilized dCas9 activator for controlling gene expression in human cell reprogramming and differentiation. Stem Cell Rep 5:448-459

Bedzhov I, Zernicka-Goetz M (2014) Self-organizing properties of mouse pluripotent cells initiate morphogenesis upon implantation. Cell 156:1032-1044

Boyer LA, Lee TI, Cole MF, Johnstone SE, Levine SS, Zucker JP, Guenther MG, Kumar RM, Murray HL, Jenner RG (2005) Core transcriptional regulatory circuitry in human embryonic stem cells. Cell 122:947-956

Brons IGM, Smithers LE, Trotter MWB, Rugg-Gunn P, Sun B, de Sousa Lopes SMC, Howlett SK, Clarkson A, Ahrlund-Richter L,
Pedersen RA (2007) Derivation of pluripotent epiblast stem cells from mammalian embryos. Nature 448:191-195

Burridge PW, Holmström A, and Wu JC (2015). Chemically defined culture and cardiomyocyte differentiation of human pluripotent stem cells. Curr Protoc Hum Genet: 21.23. 21-21.23. 15

Chambers I, Silva J, Colby D, Nichols J, Nijmeijer B, Robertson M, Vrana J, Jones K, Grotewold L, Smith A (2007) Nanog safeguards pluripotency and mediates germline development. Nature 450:1230-1234

Chang M-Y, Rhee Y-H, Yi S-H, Lee S-J, Kim R-K, Kim H, Park C-H, Lee S-H (2014) Doxycycline enhances survival and self-renewal of human pluripotent stem cells. Stem Cell Rep 3:353-364

Chavez A, Scheiman J, Vora S, Pruitt BW, Tuttle M, lyer EPR, Lin S, Kiani S, Guzman CD, Wiegand DJ (2015) Highly efficient Cas9mediated transcriptional programming. Nat Methods 12:326-328

Chen Y, Niu Y, Li Y, Ai Z, Kang Y, Shi H, Xiang Z, Yang Z, Tan T, Si W (2015) Generation of cynomolgus monkey chimeric fetuses using embryonic stem cells. Cell Stem Cell 17:116-124

Deglincerti A, Croft GF, Pietila LN, Zernicka-Goetz M, Siggia ED, Brivanlou AH (2016) Self-organization of the in vitro attached human embryo. Nature 533:251-254

DeKelver RC, Choi VM, Moehle EA, Paschon DE, Hockemeyer D, Meijsing SH, Sancak Y, Cui X, Steine EJ, Miller JC (2010) Functional genomics, proteomics, and regulatory DNA analysis in isogenic settings using zinc finger nuclease-driven transgenesis into a safe harbor locus in the human genome. Genome Res 20:1133-1142

Duggal G, Warrier S, Ghimire S, Broekaert D, Van der Jeught M, Lierman S, Deroo T, Peelman L, Van Soom A, Cornelissen R (2015) Alternative routes to induce naive pluripotency in human embryonic stem cells. Stem Cells 33:2686-2698

Gafni O, Weinberger L, Mansour AA, Manor YS, Chomsky E, BenYosef D, Kalma Y, Viukov S, Maza I, Zviran A (2013) Derivation of novel human ground state naive pluripotent stem cells. Nature 504:282-286

Genga RM, Kearns NA, Maehr R (2016) Controlling transcription in human pluripotent stem cells using CRISPR-effectors. Methods 101:36-42

Gilbert LA, Horlbeck MA, Adamson B, Villalta JE, Chen Y, Whitehead $\mathrm{EH}$, Guimaraes C, Panning B, Ploegh HL, Bassik MC (2014) Genome-scale CRISPR-mediated control of gene repression and activation. Cell 159:647-661

Gong S, Li Q, Jeter CR, Fan Q, Tang DG, Liu B (2015) Regulation of NANOG in cancer cells. Mol Carcinog 54:679-687

González F, Zhu Z, Shi Z-D, Lelli K, Verma N, Li QV, Huangfu D (2014) An iCRISPR platform for rapid, multiplexable, and inducible genome editing in human pluripotent stem cells. Cell Stem Cell 15:215-226

Hanna J, Saha K, Pando B, Van Zon J, Lengner CJ, Creyghton MP, van Oudenaarden A, Jaenisch R (2009) Direct cell reprogramming is a stochastic process amenable to acceleration. Nature 462:595-601

Hanna J, Cheng AW, Saha K, Kim J, Lengner CJ, Soldner F, Cassady JP, Muffat J, Carey BW, Jaenisch R (2010) Human embryonic stem cells with biological and epigenetic characteristics similar to those of mouse ESCs. Proc Natl Acad Sci 107:9222-9227 
Hilton IB, D'Ippolito AM, Vockley CM, Thakore PI, Crawford GE, Reddy TE, Gersbach CA (2015) Epigenome editing by a CRISPR-Cas9-based acetyltransferase activates genes from promoters and enhancers. Nat Biotechnol 33:510-517

Hockemeyer D, Soldner F, Beard C, Gao Q, Mitalipova M, DeKelver RC, Katibah GE, Amora R, Boydston EA, Zeitler B (2009) Efficient targeting of expressed and silent genes in human ESCs and iPSCs using zinc-finger nucleases. Nat Biotechnol 27:851-857

Hogan B, Costantini F, Lacy E (1986) Manipulating the mouse embryo: a laboratory manual, vol 34. Cold spring harbor laboratory, Cold Spring Harbor, NY

Hsu PD, Lander ES, Zhang F (2014) Development and applications of CRISPR-Cas9 for genome engineering. Cell 157:1262-1278

Kearns NA, Genga RMJ, Enuameh MS, Garber M, Wolfe SA, Maehr $R$ (2014) Cas9 effector-mediated regulation of transcription and differentiation in human pluripotent stem cells. Development 141:219-223

Konermann S, Brigham MD, Trevino AE, Joung J, Abudayyeh OO, Barcena C, Hsu PD, Habib N, Gootenberg JS, Nishimasu H (2014) Genome-scale transcriptional activation by an engineered CRISPR-Cas9 complex. Nature 517:583-588

Lombardo A, Cesana D, Genovese P, Di Stefano B, Provasi E, Colombo DF, Neri M, Magnani Z, Cantore A, Riso PL (2011) Sitespecific integration and tailoring of cassette design for sustainable gene transfer. Nat Methods 8:861-869

Maeder ML, Linder SJ, Cascio VM, Fu Y, Ho QH, Joung JK (2013) CRISPR RNA-guided activation of endogenous human genes. Nat Methods 10:977-979

Mandegar MA, Huebsch N, Frolov EB, Shin E, Truong A, Olvera MP, Chan AH, Miyaoka Y, Holmes K, Spencer Cl (2016) CRISPR interference efficiently induces specific and reversible gene silencing in human iPSCs. Cell Stem Cell 18:541-553

Mitsui K, Tokuzawa Y, Itoh H, Segawa K, Murakami M, Takahashi K, Maruyama M, Maeda M, Yamanaka S (2003) The homeoprotein Nanog is required for maintenance of pluripotency in mouse epiblast and ES cells. Cell 113:631-642

Ordovás L, Boon R, Pistoni M, Chen Y, Wolfs E, Guo W, Sambathkumar R, Bobis-Wozowicz S, Helsen N, Vanhove J (2015) Efficient recombinase-mediated cassette exchange in hPSCs to study the hepatocyte lineage reveals AAVS1 locusmediated transgene inhibition. Stem cell Rep 5:918-931

Qian K, Huang CL, Chen H, Blackbourn LW, Chen Y, Cao J, Yao L, Sauvey C, Du Z, Zhang SC (2014) A simple and efficient system for regulating gene expression in human pluripotent stem cells and derivatives. Stem Cells 32:1230-1238

Shahbazi MN, Jedrusik A, Vuoristo S, Recher G, Hupalowska A, Bolton V, Fogarty NME, Campbell A, Devito LG, Ilic D (2016) Self- organization of the human embryo in the absence of maternal tissues. Nature cell Biol 18:700-708

Silva J, Nichols J, Theunissen TW, Guo G, van Oosten AL, Barrandon O, Wray J, Yamanaka S, Chambers I, Smith A (2009) Nanog is the gateway to the pluripotent ground state. Cell 138:722-737

Smith JR, Maguire S, Davis LA, Alexander M, Yang F, Chandran S, Pedersen RA (2008) Robust, persistent transgene expression in human embryonic stem cells is achieved with AAVS1-targeted integration. Stem Cells 26:496-504

Taapken SM, Nisler BS, Newton MA, Sampsell-Barron TL, Leonhard KA, McIntire EM, Montgomery KD (2011) Karyotypic abnormalities in human induced pluripotent stem cells and embryonic stem cells. Nat Biotechnol 29:313-314

Takashima Y, Guo G, Loos R, Nichols J, Ficz G, Krueger F, Oxley D, Santos F, Clarke J, Mansfield W (2014) Resetting transcription factor control circuitry toward ground-state pluripotency in human. Cell 158:1254-1269

Tanenbaum ME, Gilbert LA, Qi LS, Weissman JS, Vale RD (2014) A protein-tagging system for signal amplification in gene expression and fluorescence imaging. Cell 159:635-646

Tesar PJ, Chenoweth JG, Brook FA, Davies TJ, Evans EP, Mack DL, Gardner RL, McKay RDG (2007) New cell lines from mouse epiblast share defining features with human embryonic stem cells. Nature 448:196-199

Theunissen TW, Powell BE, Wang H, Mitalipova M, Faddah DA, Reddy J, Fan ZP, Maetzel D, Ganz K, Shi L (2014) Systematic identification of culture conditions for induction and maintenance of naive human pluripotency. Cell Stem Cell 15:471-487

Ware CB, Nelson AM, Mecham B, Hesson J, Zhou W, Jonlin EC, Jimenez-Caliani AJ, Deng X, Cavanaugh C, Cook S (2014) Derivation of naive human embryonic stem cells. Proc Natl Acad Sci 111:4484-4489

Wiedenheft B, Sternberg SH, Doudna JA (2012) RNA-guided genetic silencing systems in bacteria and archaea. Nature 482:331-338

Xu X, Tao Y, Gao X, Zhang L, Li X, Zou W, Ruan K, Wang F, G-I Xu, Hu R (2016) A CRISPR-based approach for targeted DNA demethylation. Cell Discov 2:16009

Zhu Z, González F, Huangfu D (2014) The iCRISPR platform for rapid genome editing in human pluripotent stem cells. Methods Enzymol 546:215

Zhu Z, Verma N, González F, Shi Z-D, Huangfu D (2015) A CRISPR/ Cas-mediated selection-free knockin strategy in human embryonic stem cells. Stem Cell Rep 4:1103-1111 